\title{
Topological Order, Dimerization, and Spinon Deconfinement in Frustrated Spin Ladders
}

\author{
Eugene H. Kim, ${ }^{1}$ Ö. Legeza, ${ }^{2}$ and J. Sólyom ${ }^{2}$ \\ ${ }^{1}$ Department of Physics, University of Windsor, Windsor, Ontario, Canada N9B 3P4 \\ ${ }^{2}$ Research Institute for Solid State Physics and Optics, P. O. Box 49, H-1525 Budapest, Hungary
}

(Dated: October 30, 2018)

\begin{abstract}
We consider topological order and dimer order in several frustrated spin ladder models, which are related to higher dimensional models of current interest; we also address the occurrence of fractionalized phases with deconfined spinon excitations in these models. Combining results obtained with both analytic and numerical methods, we discuss how the occurrence of dimerized or fractionalized phases are dictated by the system's geometry.
\end{abstract}

PACS numbers: $71.30 .+\mathrm{h}, 71.10 . \mathrm{Fd}$

\section{INTRODUCTION}

Frustrated spin systems give rise to a wealth of interesting behavior; hence, they have attracted considerable attention. $\frac{1}{*}$ Typically, the low-lying excitations in spin systems are magnons. However, there has been particular interest in identifying fractionalized phases, where the magnon "breaks apart" into more fundamental objects. When doped, such phases would give rise to metallic states which do not fall within the Fermi liquid paradigm - the elementary excitations in these phases would not have the quantum numbers of an electron. However, finding systems which exhibit fractionalized phases has proven to be extremely challenging.

Motivated largely by Anderson's original resonating valence bond (RVB) suggestions for the high- $T_{c}$ cuprate superconductors, $\stackrel{2}{=}$ substantial effort has focussed on searching for fractionalized phases in models having short-range RVB ground states $\stackrel{3}{3}$ A related approach has been to consider dimer models, where only short-ranged valence bonds with specified dynamics are considered. $\underline{4}$ While lacking long-range order in the conventional sense, i.e., lacking a local order parameter, these short-range valence-bond ground states have a subtle form of order, namely topological order ${ }^{\frac{5}{5}}$ It is now appreciated that a precise characterization of fractionalized phases is via its topological order ${ }^{6}$ However, whether a fractionalized phase actually occurs depends strongly on the system's geometry. More specifically, the system's geometry must allow for "liquidity" in the spectrum of states ${ }^{7}$ Many systems with short-range RVB ground states do not exhibit such liquidity - they would prefer to dimerize, rather than exhibit a fractionalized phase.$\stackrel{8}{ }$

In this work, we address the occurrence of fractionalized and dimerized phases in several frustrated spin ladder models. Ladder models provide a unique testing ground, as powerful analytic and numerical techniques from one-dimensional physics can be utilized. Indeed, ladders models have allowed controlled calculations to investigate topological order $, 9.10,11$ the occurrence of dimer order, 12 and the occurrence of fractionalized excitations $\frac{13}{}$ in spin models. Furthermore, ladders models have allowed for controlled calculations demonstrating that pairing and, in particular, $d_{x^{2}-y^{2}}$ pairs could arise when these spin models are doped. $\underline{\underline{14}}$ The models we consider are related to higher-dimensional systems of current interest; investigations of the onedimensional analogs are particularly relevant, in light of recent work which showed that the mechanism giving rise to fractionalized excitations is the same in both one and two dimensions $\frac{15}{\underline{15}}$ However, besides being a testing ground and illustrating the types of possible behaviors, these ladder models are interesting in their own right, as there are a number of materials that are well described by ladder models $\underline{\underline{16}}$

In large regions of parameter space, the models we consider have short-range RVB ground states. More specifically, in large regions of parameter space these models have ground states which are continuously related to the ground states of the so-called rung-singlet phase or the Haldane phase. [Typical configurations in these ground states are shown schematically in Figs. 2]and[4in Sec. II.] While these phases have nearly identical properties, their ground states differ in a subtle way, namely in their topological order. With frustrating interactions, these states could be tuned to become degenerate, and then one has the necessary liquidity for the deconfinement of spinon excitations. However, as will be seen below, depending on the model's spatial symmetries, this liquidity and spinon deconfinement could be preempted by dimerization.

The rest of the paper is organized as follows. In Sec. II, we describe the ladder models considered in this work the cross-coupled, zigzag, and diagonal ladders - and we recall some of their known properties. In Sec. III, the exact ground states of the models are discussed (along certain lines in parameter space). Sec. IV contains a discussion of topological order in these models and some comments on the low-energy excitations. In Sec. V, we analyze the models in the limit of weak interchain couplings, using bosonization and renormalization group techniques. In Sec. VI, we present numerical results obtained via the density-matrix renormalization-group (DMRG) algorithm; we discuss these numerical results in light of the results from the previous sections. Finally, in Sec. VII we summarize and present some concluding 
remarks.

\section{THE MODELS}

We begin with two antiferromagnetic spin-1/2 Heisenberg chains, each described by the Hamiltonian

$$
H_{0}^{(i)}=\sum_{l} J_{\|} \mathbf{S}_{l}^{(i)} \cdot \mathbf{S}_{l+1}^{(i)} \quad i=1,2
$$

where $\mathbf{S}_{l}^{(i)}$ is the spin operator at site $l$ on chain $i$. We will be interested in coupling the chains together in various ways, such that the resulting models have different spatial symmetries. In this work, we will consider only antiferromagnetic couplings. We start by coupling the chains together so that the resulting models are not frustrated. We then include frustrating interactions and investigate their influence on the properties of the models.

The simplest way to couple the chains together is by

$$
H_{\perp}=\sum_{l} J_{\perp} \mathbf{S}_{l}^{(1)} \cdot \mathbf{S}_{l}^{(2)},
$$

so that the full Hamiltonian is

$$
H_{\mathrm{L}}=\sum_{i=1}^{2} H_{0}^{(i)}+H_{\perp}
$$

which is depicted in Fig. 1. We will refer to this as an ordinary ladder. This model has received considerable attention. Indeed, it can be thought of as a strip of a two-dimensional square lattice; hence, its properties have been investigated to give clues as to the physics occurring in the high- $T_{c}$ cuprate superconductors 17

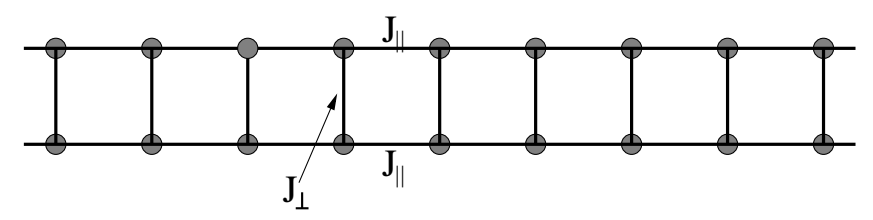

FIG. 1: The ordinary ladder.

When $J_{\perp}$ is large $\left(J_{\perp} \gg J_{\|}\right)$, the ground state is essentially a product of rung singlets with a gap to the excited states - the gap is due to the energy necessary to break a singlet bond. When $J_{\perp} \simeq J_{\|}$, it has been shown that the energy gap persists; the ground state is well described by a short-range valence-bond state, a typical configuration of which is shown in Fig. 2. It has been established that the entire region $0<J_{\perp}<\infty$ is, in fact, continuously related. 18,19 As this entire region of parameter space is related to the regime $J_{\perp} \gg J_{\|}$where the ground state is a product of rung singlets, it is often referred to as the rung-singlet phase. Incidentally, the dominance of rung-singlet bonds can be measured by determining their weight in the ground state $\rho_{s}=(1 / N) \sum_{l=1}^{N}\left\langle\Psi\left|S_{l} S_{l}^{\dagger}\right| \Psi\right\rangle$ where $|\Psi\rangle$ is the ground-state wave function and $S_{l}=$ $(1 / \sqrt{2})\left[|\uparrow\rangle_{l}^{(1)}|\downarrow\rangle_{l}^{(2)}-|\downarrow\rangle_{l}^{(1)}|\uparrow\rangle_{l}^{(2)}\right]$. A state belongs to the rung-singlet phase if $\rho_{s}>1 / 4{ }^{20}$

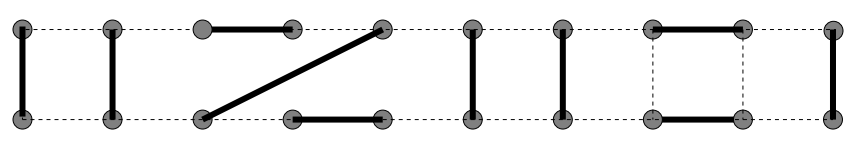

FIG. 2: A typical valence-bond configuration in the rungsinglet ground state of the two-leg ordinary Heisenberg ladder.

We also consider coupling the spins to their nextnearest neighbors on the opposite leg of the ladder. This coupling is described by

$$
H_{\mathrm{X}}=\sum_{l} J_{\mathrm{X}}\left(\mathbf{S}_{l}^{(1)} \cdot \mathbf{S}_{l+1}^{(2)}+\mathbf{S}_{l}^{(2)} \cdot \mathbf{S}_{l+1}^{(1)}\right)
$$

so that the full Hamiltonian is

$$
H_{\mathrm{C}}=\sum_{i=1}^{2} H_{0}^{(i)}+H_{\mathrm{X}} ;
$$

the resulting model is shown in Fig. 3 ,

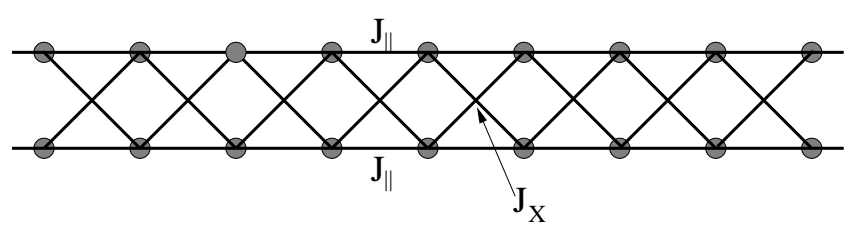

FIG. 3: Ladder with diagonal couplings.

This system is known ${ }^{21,22}$ to have a gapped spectrum of spin-1 magnons. Furthermore, the entire regime $0<$ $J_{\mathrm{X}}<\infty$ is continuously related to the Haldane phase ${ }^{23}$ of the spin- 1 chain. This can be understood by considering the point $J_{\mathrm{X}}=J_{\|}$- here, the low-energy spectrum of (5D) is equivalent to that of the $S=1$ Heisenberg spin chain. ${ }^{24.25}$ To see this, we start with the Hamiltonian of a spin-1 chain $H=J_{\|} \sum_{l} \mathbf{S}_{l} \cdot \mathbf{S}_{l+1}$, which is known to be in the Haldane phase. When the spin-1 operator on site $l$ is represented as a sum of two spin- $1 / 2$ operators, $\mathbf{S}_{l}=\mathbf{S}_{l}^{(1)}+\mathbf{S}_{l}^{(2)}$, one obtains (5) with $J_{\mathrm{X}}=J_{\|}$. Since the total spin of each rung commutes with the Hamiltonian, the eigenstates can be classified by the total spins on the rungs. It has been shown that in the low-energy part of the spectrum all rungs are in their triplet $(S=1)$ state, and hence the same Haldane gap appears in the ladder model as well. In this representation the Haldane state of the spin- 1 chain can be described rather well by short-ranged valence bonds between neighboring rungs, a typical configuration of which is shown in Fig. [4

Finally, we consider the ladder model shown in Fig. 5(a), where the interchain coupling is given by

$$
H_{\mathrm{d}}=\sum_{l} J_{2} \mathbf{S}_{2 l}^{(1)} \cdot\left(\mathbf{S}_{2 l-1}^{(2)}+\mathbf{S}_{2 l+1}^{(2)}\right) .
$$




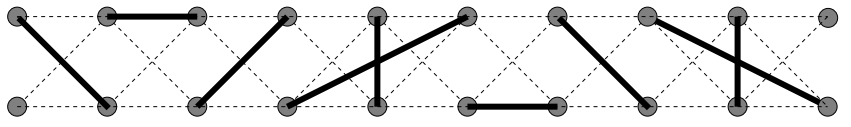

FIG. 4: A typical valence-bond configuration in the ground state of the ladder model shown in Fig. 3.

Previous work has established ${ }^{26}$ that this model is in the same universality class as the model in (5). Hence, removing half of the cross couplings in an appropriate way from Eq. (5) does not change the universality class. Part of our motivation for considering this model is because it is a representation of the " $N_{p}=2$ diagonal ladder" shown in Fig. 5(b); it is a minimal model to study diagonal stripes, which have been observed in the high- $T_{c}$ cuprate superconducting material $\mathrm{La}_{2-x} \mathrm{Sr}_{x} \mathrm{CuO}_{4}, \frac{27}{2}$ and the nickel oxides $\mathrm{La}_{2} \mathrm{NiO}_{4.125}{ }^{28}$ and $\mathrm{La}_{1-x} \mathrm{Sr}_{x} \mathrm{NiO}_{4}{ }^{29}$

(a)

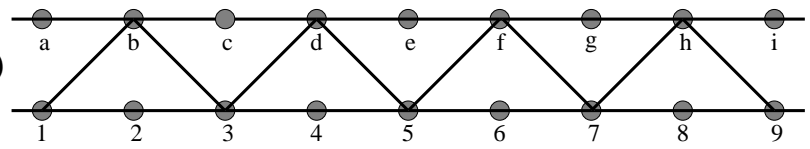

(b)

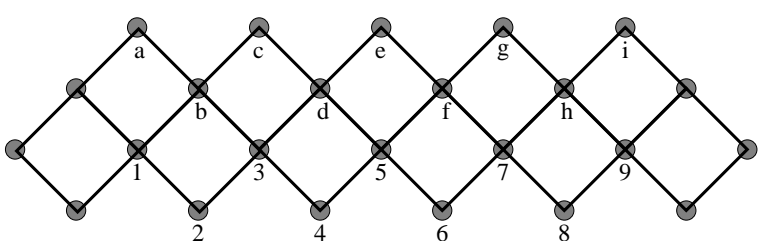

FIG. 5: (a) Ladder with a diagonal coupling between every second spin. (b) $N_{p}=2$ diagonal ladder.

As mentioned above, we are interested in the effect of frustration on these models. In what follows, we analyze three models obtained by introducing frustrating interactions to the models described above. (Again, we will consider only antiferromagnetic interchain couplings in this work.) Our motivation for doing so is because the resulting models have different spatial symmetries. As discussed below, these symmetries play a crucial role in determining the properties of the models.

\section{Cross-Coupled Ladder}

The first model we consider has both $H_{\perp}$ and $H_{\mathrm{X}}$ present simultaneously. The resulting model with Hamiltonian

$$
H_{\mathrm{CC}}=\sum_{i=1}^{2} H_{0}^{(i)}+H_{\perp}+H_{\mathrm{X}}
$$

is shown in Fig. 6. For the rest of this work, we will refer to this model as a cross-coupled ladder. Notice that this spin model is invariant under translation by a single site; it is also invariant if the legs of the ladder are interchanged: leg $1 \leftrightarrow$ leg 2 . Part of our motivation for the study of this model is its relationship to the two-dimensional model with nearest-neighbor and next-nearest-neighbor exchange couplings, often referred to as the $J_{1}-J_{2}$ model - (77) is a one-dimensional strip of this model. Furthermore, (77) can be thought of as a chain of edge-sharing tetrahedra $\stackrel{30}{\underline{\underline{3}}}$ As there are a number of materials described by spin models on corner-sharing tetrahedra, i.e., pyrochlores, 31 it is not unreasonable that materials with edge-sharing tetrahedra can be realized.

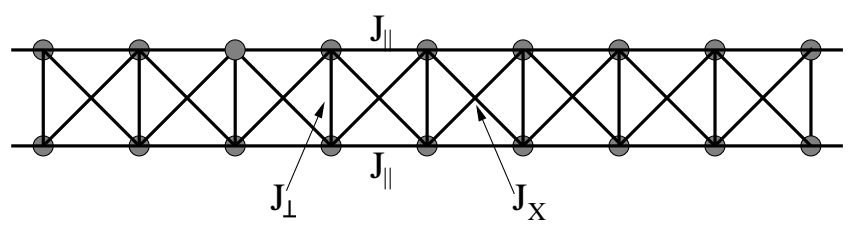

FIG. 6: The cross-coupled ladder.

The model in (7) has been investigated in a number of works, but its phase diagram and properties are still under debate. When $J_{\perp}$ is the dominant interchain coupling, the system is continuously related to the ordinary ladder (with $J_{\mathrm{X}}=0$ ); hence, the system is in the rung-singlet phase. On the other hand, when $J_{\mathrm{X}}$ is the dominant interchain coupling, the system is continuously related to Eq. (5), and the system is in the Haldane phase. For weak interchain couplings, previous analytic treatments $\frac{10}{}$ suggested a first-order transition between the rung-singlet and Haldane phases when $J_{\perp} \simeq 2 J_{\mathrm{X}}$. Numerical results on the mode $1^{11,32}$ were consistent with a first-order transition for both weak and strong interchain couplings. Recent numerical work, however, has suggested the transition is actually continuous when the interchain coupling is weak, becoming first-order only

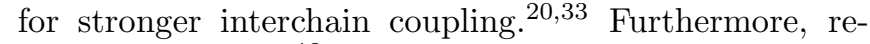
cent analytic work $\mathrm{w}^{12}$ has argued there is a spontaneously dimerized phase in between the rung-singlet and Haldane phases, rather than a direct transition between the two phases as observed in previous works.

\section{Zigzag Ladder}

The second ladder model we consider contains again both rung and diagonal couplings, but only half the diagonal couplings of the cross-coupled ladder are present, as shown in Fig. 7. This model is often referred to as a zigzag ladder; its Hamiltonian is

$$
H_{\mathrm{Z}}=\sum_{i=1}^{2} H_{0}^{(i)}+H_{\perp}+H_{\mathrm{Z}},
$$

where

$$
H_{\mathrm{z}}=\sum_{l} J_{2} \mathbf{S}_{l}^{(1)} \cdot \mathbf{S}_{l+1}^{(2)} .
$$

This spin model is invariant under translation by a single site. However, unlike the cross-coupled ladder which is 
invariant under the interchange of the legs, this model lacks that symmetry. Part of the motivation for considering this model comes from the two-dimensional triangular lattice - (8) is a one-dimensional strip of the triangular lattice. The spin-1/2 Heisenberg model on a triangular lattice has been of considerable recent interest, motivated largely in part by the discovery of the triangular lattice material $\mathrm{Cs}_{2} \mathrm{CuCl}_{4}$ and, in particular, to evidence that this material exhibits a two-dimensional fractionalized phase with deconfined spinons. ${ }^{34}$ However, besides being a toy model for understanding spin systems on a triangular lattice, this model is relevant to the quasi-one-dimensional material $\mathrm{SrCuO}_{2} \cdot 35$

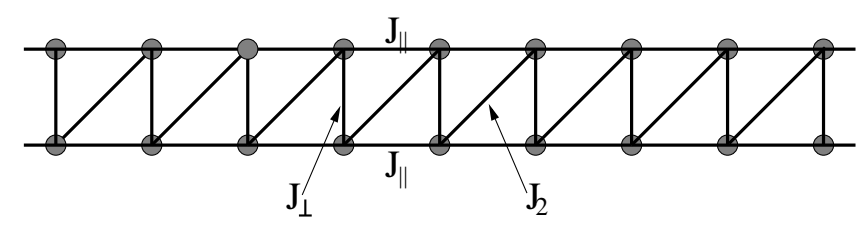

FIG. 7: The zigzag ladder model.

The properties of the zigzag ladder are well known when $J_{\perp}$ or $J_{2}$ vanishes, and also along the line $J_{\perp}=J_{2}$. When $J_{\perp}$ or $J_{2}$ vanishes, the model reduces to the ordinary ladder. When $J_{\perp}=J_{2}$, the zigzag ladder is equivalent to the spin- $1 / 2$ frustrated Heisenberg chain with nearest-neighbor coupling $J_{\perp}$ and next-nearest neighbor coupling $J_{\|}$. This chain model is known to be critical for $J_{\perp}>J_{\perp c}\left(J_{\perp c}=J_{\|} / 0.241\right), 36,37$ being in the same universality class as the antiferromagnetic spin-1/2 Heisenberg chain. The spinons of the spin-1/2 chain acquire a gap (but they remain deconfined), and the ground state becomes doubly degenerate for $J_{\perp}=J_{2}<J_{\perp c}$. 38 At the Majumdar-Ghosh (MG) point ${ }^{39} J_{\perp}=J_{2}=2 J_{\|}$, the two degenerate ground states have a simple form in the thermodynamic limit, each consisting of decoupled singlets, as shown in Fig. 8. It has been shown that these two ground states can be continuously related to the rungsinglet and Haldane phases. $\stackrel{40}{ }$ For even weaker interchain couplings $J_{\perp}=J_{2}<2 J_{\|}$, incommensurate oscillations appear in the short-range correlations. $41,42,43,44,45$

(a)

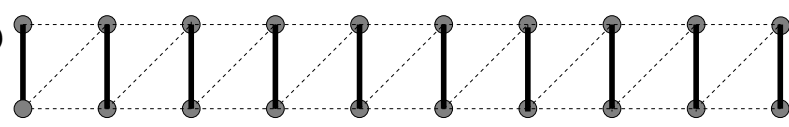

(b)

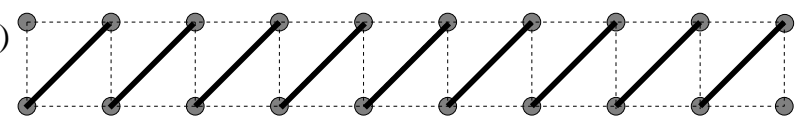

FIG. 8: The two degenerate ground states of the zigzag ladder at the Majumdar-Ghosh point.

\section{Diagonal Ladder}

Finally, we consider the model obtained when the couplings described both by (2) and (6) are present simultaneously. The model is shown in Fig. 97(a) and is described by the Hamiltonian

$$
H_{\mathrm{D}}=\sum_{i=1}^{2} H_{0}+H_{\perp}+H_{\mathrm{d}} .
$$

For the rest of this work, we will refer to this ladder model as a diagonal ladder. Unlike the previous two ladder models which were invariant under translation by a single site, (10) is invariant under translation by two sites. Furthermore, similar to the zigzag ladder, (10) is not invariant under the interchange of the legs. However, it is invariant under the combined operation of leg interchange followed by a translation by a single site. Interestingly, this ladder model can, in fact, be transformed into one which is invariant under translation by a single site, but lacking inversion symmetry. This is accomplished by interchanging the sites on every second rung of the ladder, $\mathbf{S}_{2 l}^{1} \leftrightarrow \mathbf{S}_{2 l}^{2}$; the resulting model is shown in Fig. 9. We are unaware of work which investigated the role of frustrating interactions in this model.

(a)

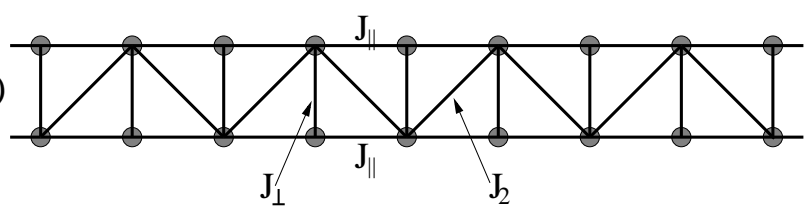

(b)

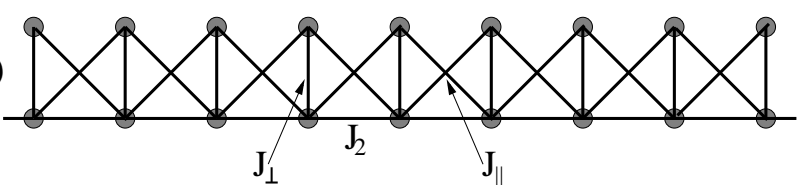

FIG. 9: (a) The diagonal ladder model. (b) Alternative representation of the diagonal ladder, obtained by interchanging sites on every second rung.

\section{EXACT GROUND STATES}

An interesting feature of the models considered in this work is that their exact ground states have a simple form for a certain regime of parameters. The key to establishing this is decomposing the system into a set of "triangles" 46 Here, we review the argument and discuss the ground states that arise.

Consider three $s=1 / 2$ spins arranged on a triangle. Suppose the spins on sites 1 and 2 are coupled by an exchange coupling $J_{1}$; these two spins are coupled to the spin on site 3 by an exchange coupling $J$. The Hamiltonian for the triangle is

$$
H_{\text {triangle }}=J_{1} \mathbf{S}_{1} \cdot \mathbf{S}_{2}+J\left(\mathbf{S}_{1}+\mathbf{S}_{2}\right) \cdot \mathbf{S}_{3} .
$$


We are interested in the case where both $J$ and $J_{1}$ are antiferromagnetic; hence, the ground state is a doublet. There are two ways of achieving this: (a) a singlet across sites 1 and 2 with a free spin- $1 / 2$ on site 3 , or (b) a triplet across sites 1 and 2 added to the spin- $1 / 2$ on site 3 to form a spin- $1 / 2$. Let $[i, j]$ denote a singlet between sites $i$ and $j$. Then, using that

$$
\left(\mathbf{S}_{i}+\mathbf{S}_{j}\right)[i, j]=0
$$

the energy of the state in (a) is found to be $E_{\mathrm{a}}=-3 J_{1} / 4$; the energy of state (b) is $E_{\mathrm{b}}=-J+J_{1} / 4$. We see that the state in (a) - the state with a singlet across sites 1 and $2-$ is the lowest-energy state if $J<J_{1}$.

Using information from the previous paragraph, we now address the ground states of the ladder models considered in this work. We begin by considering the crosscoupled ladder model. In this model, four triangles can be assigned to each rung; the Hamiltonian can be written as

$$
H_{\mathrm{CC}}=\sum_{l}\left(H_{l+}^{(1)}+H_{l-}^{(1)}+H_{l+}^{(2)}+H_{l-}^{(2)}\right)
$$

with

$H_{l+}^{(1)}=\frac{1}{4} J_{\perp} \mathbf{S}_{l}^{(1)} \cdot \mathbf{S}_{l}^{(2)}+\frac{1}{2} J_{\|} \mathbf{S}_{l}^{(1)} \cdot \mathbf{S}_{l+1}^{(1)}+\frac{1}{2} J_{\mathrm{X}} \mathbf{S}_{l}^{(2)} \cdot \mathbf{S}_{l+1}^{(1)}$, $H_{l-}^{(1)}=\frac{1}{4} J_{\perp} \mathbf{S}_{l}^{(1)} \cdot \mathbf{S}_{l}^{(2)}+\frac{1}{2} J_{\|} \mathbf{S}_{l}^{(1)} \cdot \mathbf{S}_{l-1}^{(1)}+\frac{1}{2} J_{\mathrm{X}} \mathbf{S}_{l}^{(2)} \cdot \mathbf{S}_{l-1}^{(1)}$, $H_{l+}^{(2)}=\frac{1}{4} J_{\perp} \mathbf{S}_{l}^{(1)} \cdot \mathbf{S}_{l}^{(2)}+\frac{1}{2} J_{\|} \mathbf{S}_{l}^{(2)} \cdot \mathbf{S}_{l+1}^{(2)}+\frac{1}{2} J_{\mathrm{X}} \mathbf{S}_{l}^{(1)} \cdot \mathbf{S}_{l+1}^{(2)}$, $H_{l-}^{(2)}=\frac{1}{4} J_{\perp} \mathbf{S}_{l}^{(1)} \cdot \mathbf{S}_{l}^{(2)}+\frac{1}{2} J_{\|} \mathbf{S}_{l}^{(2)} \cdot \mathbf{S}_{l-1}^{(2)}+\frac{1}{2} J_{\mathrm{X}} \mathbf{S}_{l}^{(1)} \cdot \mathbf{S}_{l-1}^{(2)} \cdot$

When $J_{\|}=J_{\mathrm{X}}$, the terms in (14) can be written in the form of (11). Then, using (12), it follows that the state

$$
|\psi\rangle=\prod_{l}[l 1, l 2]
$$

where the spins on each rung form a singlet, is an exact eigenstate of the Hamiltonian in (13) with energy

$$
E=-\frac{3}{4} J_{\perp} N
$$

We would like to determine if and when (15) is the ground state. To do so, consider each of the triangles in (14) individually. If $J_{\perp} / 2>J_{\|}$, the lowest-energy state of each triangle (when considered individually) has a singlet between the spins on the same rung. It is known that if a Hamiltonian can be written as the sum of terms, the ground-state energy cannot be smaller than the sum of the lowest energies of its constituents. For the ground state of (13), this gives the inequality

$$
E_{0} \geq-\frac{3}{4} J_{\perp} N
$$

where $N$ is the number of rungs. Hence, we see that 16) saturates the bound in (17) when $J_{\perp} / 2>J_{\|} ;(15)$ is the ground state for $J_{\|}=J_{\mathrm{X}}$ with $J_{\perp} / 2>J_{\|}, \frac{30,47}{2}$
We now consider the zigzag ladder. It can be decomposed into two triangles per rung:

$$
H_{\mathrm{Z}}=\sum_{l}\left(H_{l+}^{(2)}+H_{l-}^{(1)}\right)
$$

where

$$
\begin{aligned}
& H_{l+}^{(2)}=\frac{1}{2} J_{\perp} \mathbf{S}_{l}^{(1)} \cdot \mathbf{S}_{l}^{(2)}+J_{\|} \mathbf{S}_{l}^{(2)} \cdot \mathbf{S}_{l+1}^{(2)}+\frac{1}{2} J_{2} \mathbf{S}_{l}^{(1)} \cdot \mathbf{S}_{l+1}^{(2)}, \\
& H_{l-}^{(1)}=\frac{1}{2} J_{\perp} \mathbf{S}_{l}^{(1)} \cdot \mathbf{S}_{l}^{(2)}+J_{\|} \mathbf{S}_{l}^{(1)} \cdot \mathbf{S}_{l-1}^{(1)}+\frac{1}{2} J_{2} \mathbf{S}_{l}^{(2)} \cdot \mathbf{S}_{l-1}^{(1)} .
\end{aligned}
$$

When $J_{\|}=J_{2} / 2$, the terms in (19) can be written in the form of (11); the energy of the state in (15) saturates the lower bound in (17) for $J_{\perp} / 2>J_{\|}$. Hence, (15) is the exact ground state for $J_{\|}=J_{2} / 2$ with $J_{\perp} / 2>J_{\|} \underline{46}$ Note that another exact ground state of the zigzag ladder follows by symmetry. When $J_{\|}=J_{\perp} / 2$ and $J_{2} / 2 \geq$ $J_{\|}$the ground state is again a product of singlets, but this time they are formed diagonally between neighboring rungs: $\stackrel{46}{ }$

$$
|\psi\rangle=\prod_{l}[l 1,(l-1) 2]
$$

We now go on and consider the diagonal ladder. The diagonal ladder can be decomposed into two triangles per rung, but the triangles have different orientations for even and odd rungs:

$$
H_{\mathrm{D}}=\sum_{l}\left(H_{(2 l)+}^{(2)}+H_{(2 l)-}^{(2)}+H_{(2 l+1)+}^{(1)}+H_{(2 l+1)-}^{(1)}\right)
$$

where now

$$
\begin{aligned}
H_{(2 l)+}^{(2)}= & \frac{1}{2} J_{\perp} \mathbf{S}_{2 l}^{(1)} \cdot \mathbf{S}_{2 l}^{(2)}+J_{\|} \mathbf{S}_{2 l}^{(2)} \cdot \mathbf{S}_{2 l+1}^{(2)} \\
& +\frac{1}{2} J_{2} \mathbf{S}_{2 l}^{(1)} \cdot \mathbf{S}_{2 l+1}^{(2)}, \\
H_{(2 l)-}^{(2)}= & \frac{1}{2} J_{\perp} \mathbf{S}_{2 l}^{(1)} \cdot \mathbf{S}_{2 l}^{(2)}+J_{\|} \mathbf{S}_{2 l}^{(2)} \cdot \mathbf{S}_{2 l-1}^{(2)} \\
& +\frac{1}{2} J_{2} \mathbf{S}_{2 l}^{(1)} \cdot \mathbf{S}_{2 l-1}^{(2)}, \\
H_{(2 l+1)+}^{(1)}= & \frac{1}{2} J_{\perp} \mathbf{S}_{2 l+1}^{(1)} \cdot \mathbf{S}_{2 l+1}^{(2)}+J_{\|} \mathbf{S}_{2 l+1}^{(1)} \cdot \mathbf{S}_{2 l+2}^{(1)} \\
& +\frac{1}{2} J_{2} \mathbf{S}_{2 l+1}^{(1)} \cdot \mathbf{S}_{2 l+2}^{(2)}, \\
= & \frac{1}{2} J_{\perp} \mathbf{S}_{2 l+1}^{(1)} \cdot \mathbf{S}_{2 l+1}^{(2)}+J_{\|} \mathbf{S}_{2 l+1}^{(1)} \cdot \mathbf{S}_{2 l}^{(1)} \\
& +\frac{1}{2} J_{2} \mathbf{S}_{2 l+1}^{(2)} \cdot \mathbf{S}_{2 l}^{(1)} \cdot
\end{aligned}
$$

When $J_{\|}=J_{2} / 2$, the terms in (22) can be written in the form of (11); and thus (15) is the exact ground state for $J_{\|}=J_{2} / 2$ with $J_{\perp} / 2 \geq J_{\|}$.

\section{TOPOLOGICAL ORDER AND SPINONS IN LADDER MODELS}

¿From the discussion in Secs. II and III, the ladder models considered in this work have similar ground-state properties in extended regions of parameter space - 
their ground states are described by a collection of shortranged valence bonds, separated by a gap to the excited states. However, the ground states of these ladder models, in fact, differ in a subtle way, namely in their topological order. More specifically, the number of valence bonds crossing an arbitrary vertical line is always even in the rung-singlet phase, while the number is always odd in the Haldane phase. This can be seen explicitly in the configurations shown in Figs. 2 4, and 8. Hence a topological number $Q$ can be defined by the parity of the number of short-range valence bonds crossing an arbitrary vertical line $\stackrel{10}{=}$ This $Q$, which is either even or odd, is a good quantum number for short-range valencebond states since the Hamiltonian has finite matrix elements only between configurations with the same $Q$. For long-range valence-bond states, however, the Hamilto- nian mixes the $Q=$ even and $Q=$ odd configurations; hence, no such topological distinction is possible.

It is worth noting that for open boundary conditions $Q=$ odd ground states have spin- $1 / 2$ 's localized at the ends of the ladder, while $Q=$ even states do not. As can be seen from Figs. 4 and 8 (b), these end spins occur for topological reasons. They are analogous to the edge states in the quantum Hall effect; in general, the presence of such edge excitations is a signal of nontrivial topological order $\underline{48}$

It has also been pointed out $\underline{10}$ that the topological order of the valence bonds is related to the "hidden order" present in two-leg Heisenberg spin ladders, namely string order, analogous to the string order in antiferromagnetic spin-1 chains $\underline{49}$ This string order is detected by the two string order parameters $9,10,50$

$$
\begin{aligned}
& \mathcal{O}_{\text {odd }}^{\alpha}=-\lim _{|i-j| \rightarrow \infty}\left\langle\left(S_{i, 1}^{\alpha}+S_{i, 2}^{\alpha}\right) \exp \left(i \pi \sum_{l=i+1}^{j-1}\left(S_{l, 1}^{\alpha}+S_{l, 2}^{\alpha}\right)\right)\left(S_{j, 1}^{\alpha}+S_{j, 2}^{\alpha}\right)\right\rangle, \\
& \mathcal{O}_{\text {even }}^{\alpha}=-\lim _{|i-j| \rightarrow \infty}\left\langle\left(S_{i+1,1}^{\alpha}+S_{i, 2}^{\alpha}\right) \exp \left(i \pi \sum_{l=i+1}^{j-1}\left(S_{l+1,1}^{\alpha}+S_{l, 2}^{\alpha}\right)\right)\left(S_{j+1,1}^{\alpha}+S_{j, 2}^{\alpha}\right)\right\rangle .
\end{aligned}
$$

It was shown 19 that a slightly modified version of these string order parameters can be written in terms of the order and disorder fields of two Ising models - $\mathcal{O}_{\text {odd }}$ can be written in terms of the order fields $\sigma_{1}$ and $\sigma_{2}$; $\mathcal{O}_{\text {even }}$ can be written in terms of the disorder fields $\mu_{1}$ and $\mu_{2}$. Hence, $\mathcal{O}_{\text {odd }}$ and $\mathcal{O}_{\text {even }}$ cannot be nonzero simultaneously, as they are dual to each other. It was observed that models with $Q=$ odd ground states have $\mathcal{O}_{\text {odd }} \neq 0$, while models with $Q=$ even ground states were observed to have $\mathcal{O}_{\text {even }} \neq 0, \underline{10,11}$ Hence, the string order parameters detect the topological order of the valence bonds.

This topological order has important consequences for the excitation spectrum. In these models with shortrange valence-bond ground states, the simplest excitation is generated by breaking one of the valence bonds, promoting it to a triplet. An interesting and important question is how this excited state propagates. More specifically, does the triplet propagate coherently, or does it "break apart" so that the individual spins forming the triplet - referred to as spinons - propagate independently? In both the rung-singlet and Haldane phases, it is known that the triplet propagates coherently. The reason for this can be understood looking at Fig. 10 and counting the number of valence bonds crossing a vertical line. When a valence bond is broken in a state with $Q=$ even, if this triplet breaks apart it leaves a string of valence bonds with the "wrong" topology in the intermediate region. This gives rise to an increase in the local energy, which is proportional to the distance between the two spinons; as a result, the spinons are confined into a (gapped) spin-1 magnon. (A similar situation occurs in the $Q=$ odd Haldane phase.)

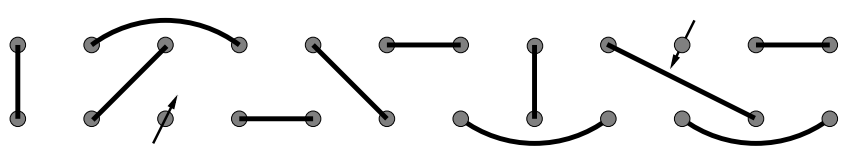

FIG. 10: Spinons separating topologically distinct regions.

¿From Fig. 10, one also sees that if the two topologically distinct ground states can be made degenerate, one can expect spinons to be deconfined. This is because the string of "wrong" valence bonds between the two spinons would not give an increase in energy. Thus, the degeneracy provides the "liquidity" necessary for spinon deconfinement. $\underline{\underline{7}}$ Indeed, as discussed in Sec. II, this is known to happen in the zigzag ladder due to the frustrating interaction. It is reasonable to expect similar phenomena could occur generically - frustrating interactions could tune the topologically distinct rung-singlet and Haldane ground states to be degenerate, so that spinons are deconfined and propagate as elementary excitations. While this expectation is reasonable, as will be discussed in detail below, there are other possible phases - namely dimerized phases - which could intervene. Moreover, we will see that the system's geometry plays a crucial role in determining whether the system may 
dimerize or not.

\section{WEAK-COUPLING ANALYSIS}

It is useful to consider the physics in the limit where the interchain coupling is weak, as controlled analytic calculations are possible. More specifically, we start with two decoupled spin-1/2 chains and consider the interchain coupling as a perturbation. Provided that there is no phase transition, the low-energy Hamiltonian deduced in the weak-coupling limit is valid even at strong coupling, albeit with renormalized parameters. This approach has been utilized in various other works; besides providing a detailed understanding of the properties of the two-leg ladder and its relation to the spin- 1 chain, $\frac{19}{19}$ it has been effective at uncovering and illucidating the phenomena that can arise in these systems. $12,13,51,52,53,54$

We begin this section by briefly describing the formalism, mainly to establish our conventions. (More detailed accounts can be found, e.g., in Ref. [55.) We go on to deduce the effective low-energy Hamiltonians for the models considered in this work, and then discuss the physics contained in these effective Hamiltonians. In particular, we discuss the role of the various irrelevant operators that arise. These operators were ignored in most previous works, but were recently argued to affect the physics

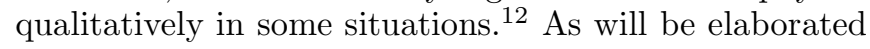
on below, quantum fluctuations can give rise to several different behaviors. To understand the physics of these quantum fluctuations, we use the renormalization group (RG) and examine the behavior of the system under a change of scale.

\section{A. Formalism}

The low-energy properties of the spin- $1 / 2$ chain are described by an SU(2), WZW model with Hamiltonian

$$
H=\frac{v}{2 \pi} \int d x\left(\mathbf{J}_{R} \cdot \mathbf{J}_{R}+\mathbf{J}_{L} \cdot \mathbf{J}_{L}\right),
$$

where the velocity $v$ is related to $J_{\|}$, and $\mathbf{J}_{R}$ and $\mathbf{J}_{L}$ are currents satisfying the $\mathrm{SU}(2)_{1}$ Kac-Moody operator product expansion (OPE)

$$
\begin{aligned}
& : J_{R}^{\alpha}(z):: J_{R}^{\beta}(w):=\frac{\delta_{\alpha, \beta}}{2(z-w)^{2}}+\frac{\mathrm{i} \epsilon^{\alpha \beta \gamma}}{2(z-w)} J_{R}^{\gamma}(w), \\
& : J_{L}^{\alpha}(\bar{z}):: J_{L}^{\beta}(\bar{w}):=\frac{\delta_{\alpha, \beta}}{2(\bar{z}-\bar{w})^{2}}+\frac{\mathrm{i} \epsilon^{\alpha \beta \gamma}}{2(\bar{z}-\bar{w})} J_{L}^{\gamma}(w),
\end{aligned}
$$

with $z=v \tau+\mathrm{i} x$ and $\bar{z}=v \tau-\mathrm{i} x$. The $\mathrm{SU}(2)_{1}$ WZW model has a single primary (matrix) field $g(z, \bar{z})$ of dimension $(1 / 4,1 / 4)$. ( $g$ is the field appearing in the $\sigma$ model representation.) Physical operators of the spin$1 / 2$ chain are given by combinations of the components $g$ - the staggered magnetization $n^{\alpha}(z, \bar{z})$ and the dimerization $\epsilon(z, \bar{z})$ :

$$
n^{\alpha}(z, \bar{z})=\operatorname{Tr}\left[\sigma^{\alpha} g(z, \bar{z})\right], \quad \epsilon(z, \bar{z})=\operatorname{Tr}[g(z, \bar{z})],
$$

where the $\left\{\sigma^{\alpha}\right\}$ are Pauli matrices. The $n^{\alpha}$ and $\epsilon$ fields have leading short-distance behavior

$$
\begin{aligned}
: n^{\alpha}(z):: n^{\beta}(w) & :=\frac{\delta_{\alpha \beta}}{|z-w|}, \\
: \epsilon(z):: \epsilon(w) & :=\frac{1}{|z-w|}, \\
: n^{\alpha}(z):: \epsilon(w): & =0 .
\end{aligned}
$$

Furthermore, being linear combinations of the components of $g$, their OPE's with the currents are

$$
\begin{aligned}
& : J_{R}^{\alpha}(z):: n^{\beta}(w):=\frac{\mathrm{i}}{2(z-w)}\left[\epsilon^{\alpha \beta \gamma} n^{\gamma}(w)-\delta_{\alpha \beta} \epsilon(w)\right] \\
& : J_{L}^{\alpha}(z):: n^{\beta}(w):=\frac{\mathrm{i}}{2(\bar{z}-\bar{w})}\left[\epsilon^{\alpha \beta \gamma} n^{\gamma}(w)+\delta_{\alpha \beta} \epsilon(w)\right] \\
& : J_{R}^{\alpha}(z):: \epsilon(w):=\frac{\mathrm{i}}{2(z-w)} n^{\alpha}(w), \\
& : J_{L}^{\alpha}(z):: \epsilon(w):=\frac{-\mathrm{i}}{2(\bar{z}-\bar{w})} n^{\alpha}(w) .
\end{aligned}
$$

[In Eqs. (27) and (28), $n^{\alpha}(z) \equiv n^{\alpha}(z, \bar{z})$ and $\epsilon(z) \equiv$ $\epsilon(z, \bar{z})$.]

At low energies, the spin operator on chain $i, \mathbf{S}_{l}^{(i)}$, can be written as

$$
\frac{\mathbf{S}_{l}^{(i)}}{a}=\frac{1}{2 \pi}\left(\mathbf{J}_{i R}(x)+\mathbf{J}_{i L}(x)\right)+(-1)^{l} \frac{M}{2 \pi a^{1 / 2}} \mathbf{n}_{i}(x),
$$

where $a$ is an ultraviolet regulator $(a \sim$ the lattice spacing), and $M$ is a nonuniversal $\mathcal{O}(1)$ constant. In what follows, we will need to know the behavior of the fields upon translation by a single site. ¿From the invariance of the spin operator $\mathbf{S}_{l}^{(i)}$ under translation we deduce how $\mathbf{J}_{i R}(x), \mathbf{J}_{i L}(x)$, and $\mathbf{n}_{i}(x)$ transform. Then, as all of the terms on the right-hand-side of (28) must transform in the same way upon translation, we deduce how $\epsilon_{i}(x)$ transforms. Hence, we arrive at

$$
\begin{aligned}
\mathbf{J}_{i R / L}(x) & \rightarrow \mathbf{J}_{i R / L}(x), \\
\mathbf{n}_{i}(x) \rightarrow-\mathbf{n}_{i}(x), & \epsilon_{i}(x) \rightarrow-\epsilon_{i}(x) .
\end{aligned}
$$

\section{B. Operators and Phases: Topological Order, Dimer Order, and Deconfined Spinons}

As described above, starting from two spin- $1 / 2$ chains, we are interested in the fate of the system upon turning on an interchain coupling. In this subsection, we discuss the various operators that can arise in the low-energy Hamiltonian(s); we discuss the physics that these operators give rise to. 
The low-energy Hamiltonians are dictated by symmetry. The models we are considering are all SU(2) symmetric; hence, all operators appearing must transform as $\mathrm{SU}(2)$ scalars. Therefore, if only relevant and marginal operators are considered, the operators

$$
\begin{aligned}
& \epsilon_{1} \text { and } \epsilon_{2}, \quad \epsilon_{1} \epsilon_{2}, \quad \mathbf{n}_{1} \cdot \mathbf{n}_{2}, \quad \mathbf{n}_{1} \partial_{x} \mathbf{n}_{2}, \quad \epsilon_{1} \partial_{x} \epsilon_{2}, \\
& \left(\mathbf{J}_{1 R}+\mathbf{J}_{1 L}\right) \cdot \mathbf{n}_{2}, \quad\left(\mathbf{J}_{2 R}+\mathbf{J}_{2 L}\right) \cdot \mathbf{n}_{1}, \\
& \left(\mathbf{J}_{1 R} \cdot \mathbf{J}_{2 L}+\mathbf{J}_{2 R} \cdot \mathbf{J}_{1 L}\right), \quad\left(\mathbf{J}_{1 R} \cdot \mathbf{J}_{1 L}+\mathbf{J}_{2 L} \cdot \mathbf{J}_{2 R}\right)
\end{aligned}
$$

have the potential of appearing in the low-energy Hamiltonians. Furthermore, we will not consider the terms $\mathbf{J}_{1 R} \cdot \mathbf{J}_{2 R}$ and $\mathbf{J}_{1 L} \cdot \mathbf{J}_{2 L}$. Even though these terms have dimension two (and, hence, are marginal), they couple excitations moving in the same direction on both chains and give rise only to small quantitative corrections. As discussed below, other symmetries of the various models will further restrict the operators allowed in their lowenergy Hamiltonians.

The physics is determined by the operator which flows to strong coupling first under the RG. When the $\mathbf{n}_{1} \cdot \mathbf{n}_{2}$ term determines the physics, the resulting phases have gapped magnons with spinons being confined. ${ }^{19}$ [The $\mathbf{n}_{1} \cdot \mathbf{n}_{2}$ term, in fact, gives rise to a strong confining potential which binds together spinons from the two chains to form a gapped magnon.] Furthermore, these phases have topological order; information about this topological order is contained in the sign of the $\mathbf{n}_{1} \cdot \mathbf{n}_{2}$ term's coefficient. Another possibility is if the physics is determined by the $\left(\mathbf{J}_{1 R} \cdot \mathbf{J}_{2 L}+\mathbf{J}_{2 R} \cdot \mathbf{J}_{1 L}\right)$ term. When this occurs, it has been shown that the resulting phase is a fractionalized phase with deconfined spinons. ${ }^{13}$ Finally, if the physics is determined by the $\epsilon_{1}$ and $\epsilon_{2}$ or $\epsilon_{1} \epsilon_{2} \operatorname{term}(\mathrm{s})$, the result is a spontaneously dimerized phase.

There are other operators in Eq. (31). However, these operators do not determine the phases that arise, though they modify the properties within a particular phase. In particular, $\left(\mathbf{J}_{1 R} \cdot \mathbf{J}_{1 L}+\mathbf{J}_{2 L} \cdot \mathbf{J}_{2 R}\right)$ is the marginally irrelevant operator present in the two spin- $1 / 2$ chains. 56 Furthermore, $\mathbf{n}_{1} \cdot \partial_{x} \mathbf{n}_{2}$ and $\epsilon_{1} \partial_{x} \epsilon_{2}$ are believed to give rise to incommensurate correlations $\frac{13,54}{}$ Finally, as will be seen below, the $\left(\mathbf{J}_{1 R}+\mathbf{J}_{1 L}\right) \cdot \mathbf{n}_{2}$ and $\left(\mathbf{J}_{2 R}+\mathbf{J}_{2 L}\right) \cdot \mathbf{n}_{1}$ are always subleading to $\mathbf{n}_{1} \cdot \mathbf{n}_{2}$ and $\epsilon_{1} \epsilon_{2}$ and, hence, do not determine the phases that arise.

\section{Effective Low-Energy Hamiltonians}

Here we deduce the effective low-energy Hamiltonians for the frustrated ladder models considered in this work. We then derive renormalization group ( $R G$ ) equations describing how the parameters in the low-energy Hamiltonian evolve under a change of scale. Finally, we derive the initial values of the parameters from the microscopic models in the limit of weak interchain coupling.

\section{Cross-Coupled Ladder}

Besides being SU(2)-symmetric, the cross-coupled ladder is invariant under translation by one site and also inversion about the center of the ladder: leg $1 \leftrightarrow \operatorname{leg} 2$. Translation by one site forbids $\epsilon_{1}$ and $\epsilon_{2}$ from appearing alone; it also forbids the operators $\left(\mathbf{J}_{1 R}+\mathbf{J}_{1 L}\right) \cdot \mathbf{n}_{2}$ and $\left(\mathbf{J}_{2 R}+\mathbf{J}_{2 L}\right) \cdot \mathbf{n}_{1}$. Inversion about the center forces operators from leg 1 and leg 2 to appear symmetrically; in particular, it forbids the operators $\mathbf{n}_{1} \partial_{x} \mathbf{n}_{2}$ and $\epsilon_{1} \partial_{x} \epsilon_{2}$ from appearing. These symmetries constrain the effective low-energy Hamiltonian to have the form

$$
\begin{aligned}
& H=\int d x\left[\gamma\left(\mathbf{J}_{1 R} \cdot \mathbf{J}_{1 L}+\mathbf{J}_{2 L} \cdot \mathbf{J}_{2 R}\right)\right. \\
& \left.+\lambda\left(\mathbf{J}_{1 R} \cdot \mathbf{J}_{2 L}+\mathbf{J}_{1 L} \cdot \mathbf{J}_{2 R}\right)+\frac{g}{a} \mathbf{n}_{1} \cdot \mathbf{n}_{2}+\frac{\beta}{a} \epsilon_{1} \epsilon_{2}\right] .
\end{aligned}
$$

To understand the physics contained in (32), we use the $\mathrm{RG}$ and investigate the behavior under a transformation of scale. Using the OPE's in (25), (27), and (28), we deduce the $\mathrm{RG}$ equations for the parameters to be

$$
\begin{aligned}
\frac{d \gamma}{d l} & =\gamma^{2}-\frac{1}{2} g^{2}+\frac{1}{2} \beta^{2}, \\
\frac{d \lambda}{d l} & =\lambda^{2}+g^{2}-g \beta, \\
\frac{d g}{d l} & =g-\frac{1}{2} \gamma g+\lambda g-\frac{1}{2} \lambda \beta, \\
\frac{d \beta}{d l} & =\beta+\frac{3}{2} \gamma \beta-\frac{3}{2} \lambda g .
\end{aligned}
$$

¿From the structure of the RG equations, we see that if either $g$ or $\beta$ is nonzero, both $\mathbf{n}_{1} \cdot \mathbf{n}_{2}$ and $\epsilon_{1} \epsilon_{2}$ will be generated upon renormalization. However, if both $g=0$ and $\beta=0$, these operators will not appear. Hence, one would expect the relevant $\mathbf{n}_{1} \cdot \mathbf{n}_{2}$ or $\epsilon_{1} \epsilon_{2}$ terms to determine the physics under most situations. If both of these terms are suppressed, the physics would be determined by the marginally relevant $\left(\mathbf{J}_{1 R} \cdot \mathbf{J}_{2 L}+\mathbf{J}_{1 L} \cdot \mathbf{J}_{2 R}\right)$.

To deduce the values of the parameters in (32), we insert (29) into (2) and (4); we obtain

$$
\begin{aligned}
H_{\perp}+H_{\mathrm{X}} & =\int \frac{d x}{(2 \pi)^{2}}\left[\left(J_{\perp}-2 J_{\mathrm{X}}\right) \frac{M^{2}}{a} \mathbf{n}_{1} \cdot \mathbf{n}_{2}\right. \\
& -2 J_{\mathrm{X}} \frac{M^{2}}{a} \sum_{m=1}^{\infty} \frac{a^{2 m}}{(2 m) !} \mathbf{n}_{1} \partial_{x}^{2 m} \mathbf{n}_{2} \\
& \left.+\left(J_{\perp}+2 J_{\mathrm{X}}\right)\left(\mathbf{J}_{1 R} \cdot \mathbf{J}_{2 L}+\mathbf{J}_{1 L} \cdot \mathbf{J}_{2 R}\right)\right] .
\end{aligned}
$$

A lattice Hamiltonian gives rise to a continuum field theory with an infinite number of operators 56 Here, besides $\mathbf{n}_{1} \cdot \mathbf{n}_{2}$ and $\left(\mathbf{J}_{1 R} \cdot \mathbf{J}_{2 L}+\mathbf{J}_{1 L} \cdot \mathbf{J}_{2 R}\right)$, an infinite number of irrelevant operators of the form $\mathbf{n}_{1} \cdot \partial_{x}^{2 m} \mathbf{n}_{2}$ appear. ¿From Eq. (34), the relevant operator $\mathbf{n}_{1} \cdot \mathbf{n}_{2}$ determines the physics for generic values of $J_{\|}$and $J_{\perp}$. When the $\mathbf{n}_{1} \cdot \mathbf{n}_{2}$ term is suppressed, it appears the physics is determined by the $\left(\mathbf{J}_{1 R} \cdot \mathbf{J}_{2 L}+\mathbf{J}_{1 L} \cdot \mathbf{J}_{2 R}\right)$ term. However, things are 
more subtle - the irrelevant $\mathbf{n}_{1} \cdot \partial_{x}^{2 m} \mathbf{n}_{2}$ can generate the relevant $\mathbf{n}_{1} \cdot \mathbf{n}_{2}$ and $\epsilon_{1} \epsilon_{2}$ when integrated out. 12 Indeed, focussing on the leading irrelevant operator $\mathbf{n}_{1} \cdot \partial_{x}^{2} \mathbf{n}_{2}$, the term

$$
\begin{gathered}
\left(J_{\perp}+2 J_{\mathrm{X}}\right) \int \frac{d^{2} x_{1}}{(2 \pi)^{2}}\left(\mathbf{J}_{1 R} \cdot \mathbf{J}_{2 L}+\mathbf{J}_{1 L} \cdot \mathbf{J}_{2 R}\right) \\
\times J_{\mathrm{X}} M^{2} a \int \frac{d^{2} x_{2}}{(2 \pi)^{2}} \mathbf{n}_{1} \cdot \partial_{x}^{2} \mathbf{n}_{2}
\end{gathered}
$$

appears in the partition function at second order. Using the OPE's in (28),

$$
\frac{J_{\mathrm{X}}\left(J_{\perp}+2 J_{\mathrm{X}}\right) M^{2}}{(2 \pi)^{2} a}\left[\frac{1}{2} \mathbf{n}_{1} \cdot \mathbf{n}_{2}-\frac{3}{4} \epsilon_{1} \epsilon_{2}\right]
$$

is generated in the low-energy Hamiltonian. Hence, to leading order we deduce

$$
\begin{aligned}
& \lambda=\frac{J_{\perp}+2 J_{\mathrm{X}}}{(2 \pi)^{2}}, \quad \beta=-\frac{3}{8 \pi} \frac{\left(J_{\perp}+2 J_{\mathrm{X}}\right) J_{\mathrm{X}} M^{2}}{(2 \pi)^{2}} \\
& g=\frac{\left(J_{\perp}-2 J_{\mathrm{X}}\right) M^{2}}{(2 \pi)^{2}}+\frac{J_{\mathrm{X}} M^{2}}{4 \pi} \frac{J_{\perp}+2 J_{\mathrm{X}}}{(2 \pi)^{2}}
\end{aligned}
$$

As mentioned above, the relevant operator $\mathbf{n}_{1} \cdot \mathbf{n}_{2}$ determines the physics for generic values of $J_{\|}$and $J_{\perp}$. Now when $g \rightarrow 0$ in (37), the relevant $\epsilon_{1} \epsilon_{2}$ is present (from integrating out the irrelevant operators); one may expect it to determine the physics. However, its coefficient is significantly smaller than the $\left(\mathbf{J}_{1 R} \cdot \mathbf{J}_{2 L}+\mathbf{J}_{1 L} \cdot \mathbf{J}_{2 R}\right)$ term. Therefore, a subtle competition between the two interactions should be expected. This competition will be investigated in detail in our numerical calculations.

\section{Zigzag Ladder}

Like the cross-coupled ladder, the zigzag ladder is invariant under translation by a single site. However, it lacks the symmetry of inversion about the center of the ladder. Hence, the operators from leg 1 and leg 2 do not have to appear symmetrically - now the operators $\mathbf{n}_{1} \partial_{x} \mathbf{n}_{2}$ and $\epsilon_{1} \partial_{x} \epsilon_{2}$ are allowed. The effective low-energy Hamiltonian has the form

$$
\begin{aligned}
& H=\int d x\left[\gamma\left(\mathbf{J}_{1 R} \cdot \mathbf{J}_{1 L}+\mathbf{J}_{2 L} \cdot \mathbf{J}_{2 R}\right)\right. \\
& +\lambda\left(\mathbf{J}_{1 R} \cdot \mathbf{J}_{2 L}+\mathbf{J}_{1 L} \cdot \mathbf{J}_{2 R}\right) \\
& \left.+\frac{g}{a} \mathbf{n}_{1} \cdot \mathbf{n}_{2}+\frac{\beta}{a} \epsilon_{1} \epsilon_{2}+g_{1} \mathbf{n}_{1} \cdot \partial_{x} \mathbf{n}_{2}+\beta_{1} \epsilon_{1} \partial_{x} \epsilon_{2}\right]
\end{aligned}
$$

As with the cross-coupled ladder, we investigate the physics of Eq. (38) using the RG. Using the OPE's, we deduce the $R G$ equations for the parameters to be

$$
\begin{aligned}
\frac{d \gamma}{d l} & =\gamma^{2}-\frac{1}{2} g^{2}+\frac{1}{2} \beta^{2}+\frac{1}{4} g_{1}^{2}-\frac{1}{4} \beta_{1}^{2}, \\
\frac{d \lambda}{d l} & =\lambda^{2}+g^{2}-g \beta+\frac{1}{2} g_{1}^{2}-\frac{1}{2} g_{1} \beta_{1}, \\
\frac{d g}{d l} & =g-\frac{1}{2} \gamma g+\lambda g-\frac{1}{2} \lambda \beta, \\
\frac{d \beta}{d l} & =\beta+\frac{3}{2} \gamma \beta-\frac{3}{2} \lambda g \\
\frac{d g_{1}}{d l} & =-\frac{1}{2} \gamma g_{1}+\lambda g_{1}-\frac{1}{2} \lambda \beta_{1}, \\
\frac{d \beta_{1}}{d l} & =\frac{3}{2} \gamma \beta_{1}-\frac{3}{2} \lambda g_{1} .
\end{aligned}
$$

As with the cross-coupled ladder, $\mathbf{n}_{1} \cdot \mathbf{n}_{2}$ and $\epsilon_{1} \epsilon_{2}$ will be generated upon renormalization unless both $g=0$ and $\beta=0$. Similarly, $\mathbf{n}_{1} \cdot \partial \mathbf{n}_{2}$ and $\epsilon_{1} \partial \epsilon_{2}$ will appear unless both $g_{1}=0$ and $\beta_{1}=0$. Hence, as with the cross-coupled ladder, one would expect the $\mathbf{n}_{1} \cdot \mathbf{n}_{2}$ or $\epsilon_{1} \epsilon_{2}$ terms to determine the physics under most situations; if they are suppressed, the physics would be determined by $\left(\mathbf{J}_{1 R} \cdot \mathbf{J}_{2 L}+\mathbf{J}_{1 L} \cdot \mathbf{J}_{2 R}\right)$.

To deduce the values of the parameters in Eq. (38), we insert Eq. (29) into Eqs. (2) and (8); we obtain

$$
\begin{aligned}
& H_{\perp}+H_{\mathrm{z}}=\int \frac{d x}{(2 \pi)^{2}}\left\{\left(J_{\perp}-J_{2}\right) \frac{M^{2}}{a} \mathbf{n}_{1} \cdot \mathbf{n}_{2}\right. \\
& +\frac{M^{2}}{a} \sum_{m=1}^{\infty} \frac{1}{m !}\left(\frac{a}{2}\right)^{m}\left[J_{\perp}(-1)^{m}-J_{2}\right] \mathbf{n}_{1} \partial_{x}^{m} \mathbf{n}_{2} \\
& \left.\quad+\left(J_{\perp}+J_{2}\right)\left(\mathbf{J}_{1 R} \cdot \mathbf{J}_{1 L}+\mathbf{J}_{2 L} \cdot \mathbf{J}_{2 R}\right)\right\} .
\end{aligned}
$$

As we saw with the cross-coupled ladder, the lattice Hamiltonian gives rise to an infinite number of operators in the field theory. Similar to the cross-coupled ladder, the relevant $\mathbf{n}_{1} \cdot \mathbf{n}_{2}$ determines the physics for generic values of $J_{\|}$and $J_{\perp}$. Furthermore, when the $\mathbf{n}_{1} \cdot \mathbf{n}_{2}$ term is suppressed, the irrelevant operators may generate terms which determine the physics. Interestingly, for this model all the terms of the form $\mathbf{n}_{1} \cdot \partial_{x}^{2 m} \mathbf{n}_{2}$ have their coefficient proportional to $\left(J_{\perp}-J_{2}\right)$. Hence, all the operators of the form $\mathbf{n}_{1} \cdot \partial_{x}^{2 m} \mathbf{n}_{2}$ are fine-tuned away along the line $J_{\perp}=J_{2}$; only terms of the form $\mathbf{n}_{1} \cdot \partial_{x}^{2 m+1} \mathbf{n}_{2}$ are present. As there are only derivatives of odd power, they are unable to generate terms like $\mathbf{n}_{1} \cdot \mathbf{n}_{2}$ or $\epsilon_{1} \epsilon_{2}$. Therefore, for this model the irrelevant operators do not change the physics (though they give rise to small quantitative changes, e.g., in the size of energy gaps); to leading order we deduce the parameters in Eq. (38) to be

$$
\begin{gathered}
\lambda=\frac{J_{\perp}+J_{2}}{(2 \pi)^{2}}, \quad g=\frac{\left(J_{\perp}-J_{2}\right) M^{2}}{(2 \pi)^{2}}, \quad \beta=0, \\
g_{1}=-\frac{\left(J_{\perp}+J_{2}\right) M^{2}}{2(2 \pi)^{2}}, \quad \beta_{1}=0 .
\end{gathered}
$$




\section{Diagonal Ladder}

Like the previous two models, the diagonal ladder has translation invariance. However, unlike the cross-coupled and zigzag ladders which are invariant under translation by a single site, the diagonal ladder is invariant under translation by two sites. Furthermore, while this model is not invariant under inversion about the center of the ladder (leg $1 \leftrightarrow \operatorname{leg} 2$ ), it is invariant under inversion compounded by translation by one site. Due to the invariance under translation by two sites, the operators $\epsilon_{1}, \epsilon_{2}$, $\left(\mathbf{J}_{1 R}+\mathbf{J}_{1 L}\right) \cdot \mathbf{n}_{2}$ and $\left(\mathbf{J}_{2 R}+\mathbf{J}_{2 L}\right) \cdot \mathbf{n}_{1}$ are now allowed. However, the symmetry of inversion compounded by translation by one site constrains them to appear in the combination $\left(\epsilon_{1}-\epsilon_{2}\right)$ and $\left[\left(\mathbf{J}_{1 R}+\mathbf{J}_{1 L}\right) \cdot \mathbf{n}_{2}-\left(\mathbf{J}_{2 R}+\mathbf{J}_{2 L}\right) \cdot \mathbf{n}_{1}\right]$. Hence, the effective low-energy Hamiltonian has the form

$$
\begin{aligned}
& H=\int d x\left[\frac{\alpha}{a^{3 / 2}}\left(\epsilon_{1}-\epsilon_{2}\right)+\gamma\left(\mathbf{J}_{1 R} \cdot \mathbf{J}_{1 L}+\mathbf{J}_{2 L} \cdot \mathbf{J}_{2 R}\right)\right. \\
& +\lambda\left(\mathbf{J}_{1 R} \cdot \mathbf{J}_{2 L}+\mathbf{J}_{1 L} \cdot \mathbf{J}_{2 R}\right)+\frac{g}{a} \mathbf{n}_{1} \cdot \mathbf{n}_{2}+\frac{\beta}{a} \epsilon_{1} \epsilon_{2} \\
& \left.+\frac{\kappa}{a^{1 / 2}}\left[\left(\mathbf{J}_{1 R}+\mathbf{J}_{1 L}\right) \cdot \mathbf{n}_{2}-\left(\mathbf{J}_{2 R}+\mathbf{J}_{2 L}\right) \cdot \mathbf{n}_{1}\right]\right]
\end{aligned}
$$

As before, we use the OPE's to deduce the RG equations for the parameters; we find

$$
\begin{aligned}
\frac{d \gamma}{d l} & =\gamma^{2}-\frac{1}{2} g^{2}+\frac{1}{2} \beta^{2}+\frac{1}{2} \alpha^{2}, \\
\frac{d \lambda}{d l} & =\lambda^{2}+g^{2}-g \beta, \\
\frac{d g}{d l} & =g-\frac{1}{2} \gamma g+\lambda g-\frac{1}{2} \lambda \beta-\kappa^{2}, \\
\frac{d \beta}{d l} & =\beta+\frac{3}{2} \gamma \beta-\frac{3}{2} \lambda g+\alpha^{2}-\frac{3}{2} \kappa^{2}, \\
\frac{d \alpha}{d l} & =\frac{3}{2} \alpha+\frac{3}{4} \gamma \alpha+\beta \alpha, \\
\frac{d \kappa}{d l} & =\frac{1}{2} \kappa-\frac{1}{4} \gamma \kappa+\frac{1}{4} \lambda \kappa-g \kappa+\frac{1}{2} \beta \kappa .
\end{aligned}
$$

For this ladder model, the operator $\left(\epsilon_{1}-\epsilon_{2}\right)$ is allowed; if its initial coefficient is zero, this term will never be generated. Furthermore, we must have $\alpha=0, g=0$, $\beta=0$, and $\kappa=0$ in order for all the relevant operators to be banished. In particular, if any of these are nonzero, the $\mathbf{n}_{1} \cdot \mathbf{n}_{2}$ and $\epsilon_{1} \epsilon_{2}$ terms will be generated.

To deduce the values of the parameters in Eq. (42), we insert (29) into (10); we obtain

$$
\begin{aligned}
& H_{\perp}+H_{\mathrm{d}}=\int \frac{d x}{(2 \pi)^{2}}\left\{\left(J_{\perp}-J_{2}\right) \frac{M^{2}}{a} \mathbf{n}_{1} \cdot \mathbf{n}_{2}\right. \\
& -J_{2} \frac{M^{2}}{a} \sum_{m=1}^{\infty} \frac{a^{2 m}}{(2 m) !} \mathbf{n}_{1} \cdot \partial_{x}^{2 m} \mathbf{n}_{2} \\
& +\left(J_{\perp}+J_{2}\right)\left(\mathbf{J}_{1 R} \cdot \mathbf{J}_{1 L}+\mathbf{J}_{2 L} \cdot \mathbf{J}_{2 R}\right) \\
& +\frac{J_{2} M}{a^{1 / 2}}\left[\left(\mathbf{J}_{1 R}+\mathbf{J}_{1 L}\right) \cdot \mathbf{n}_{2}-\left(\mathbf{J}_{2 R}+\mathbf{J}_{2 L}\right) \cdot \mathbf{n}_{1}\right] \\
& +\frac{J_{2} M}{a^{1 / 2}} \sum_{m=1}^{\infty} \frac{a^{2 m}}{(2 m) !}\left[\left(\mathbf{J}_{1 R}+\mathbf{J}_{1 L}\right) \cdot \partial_{x}^{2 m} \mathbf{n}_{2}\right. \\
& \left.\left.\quad-\left(\mathbf{J}_{2 R}+\mathbf{J}_{2 L}\right) \cdot \partial_{x}^{2 m} \mathbf{n}_{1}\right]\right\} .
\end{aligned}
$$

Interestingly, even though the term $\left(\epsilon_{1}-\epsilon_{2}\right)$ is allowed, its coefficient is zero. However, the relevant $\left[\left(\mathbf{J}_{1 R}+\mathbf{J}_{1 L}\right)\right.$. $\left.\mathbf{n}_{2}-\left(\mathbf{J}_{2 R}+\mathbf{J}_{2 L}\right) \cdot \mathbf{n}_{1}\right]$ is always present; as mentioned above, it generates both the $\mathbf{n}_{1} \cdot \mathbf{n}_{2}$ and $\epsilon_{1} \epsilon_{2}$ terms. Hence, for this model the irrelevant operators give rise only to small quantitative corrections; the physics that can occur is already contained in the relevant operators already present. Therefore, to leading order we deduce the values of the parameters in (42) to be

$$
\begin{gathered}
\lambda=\frac{J_{\perp}+J_{2}}{(2 \pi)^{2}}, \quad g=\frac{\left(J_{\perp}-J_{2}\right) M^{2}}{(2 \pi)^{2}}, \quad \beta=0, \\
\kappa=\frac{J_{2} M}{(2 \pi)^{2}}, \quad \alpha=0 .
\end{gathered}
$$

As mentioned above, the zigzag ladder is expected to have a fractionalized phase when $g \rightarrow 0$; one can expect a subtle competition between $\epsilon_{1} \epsilon_{2}$ and $\left(\mathbf{J}_{1 R} \cdot \mathbf{J}_{2 L}+\mathbf{J}_{1 L} \cdot \mathbf{J}_{2 R}\right)$ in the cross-coupled ladder. For this ladder model, as $\epsilon_{1} \epsilon_{2}$ is generated by the relevant $\left[\left(\mathbf{J}_{1 R}+\mathbf{J}_{1 L}\right) \cdot \mathbf{n}_{2}-\left(\mathbf{J}_{2 R}+\right.\right.$ $\left.\mathbf{J}_{2 L}\right) \cdot \mathbf{n}_{1}$ ] term, it is reasonable to expect a regime of parameter space where $\epsilon_{1} \epsilon_{2}$ flows to strong coupling first and the system dimerizes.

\section{NUMERICAL RESULTS AND DISCUSSION}

In this section, we present our numerical results for the phase diagrams of the models introduced in Sec. II; we discuss them in light of the analytical results presented in the previous sections.

\section{A. Numerical Method}

The numerical calculations have been performed on finite ladders with open boundary condition (OBC) using the DMRG algorithm ${ }^{59}$ with the dynamic block-state selection (DBSS) approach ${ }^{60,61}$ We have set the threshold value of the quantum information loss $\chi$ to $10^{-8}$ and the minimum number of block states $M_{\min }$ to 64 . All relevant eigenstates have been targeted independently using four 
to six DMRG sweeps until the entropy sum rule has been satisfied. The accuracy of the Davidson diagonalization routine has been set to $10^{-7}$.

Recently, it has been shown that quantum phase transitions (QPTs) can be conveniently studied by calculating some measure of entanglement $62,63,64,65,66,67,68,69,70,71,72$ In particular, the von Neumann entropy of a block containing a finite number of neighboring sites often gives a clear indication of a QPT, as anomalies appear in these quantities at the transition: the entropy exhibits a jump at a first-order transition or develops a cusp (with increasing $N$ ) at a continuous transition. It should be noted, however, that depending on how a first-order transition is realized, one might have difficulty distinguishing it from a continuous transition. More specifically, if the two levels corresponding to the different ground states are already orthogonal in a finite-sized system, the entropy of a block will exhibit a jump when the two levels cross (in a finite-sized system). However, if the two levels are not orthogonal in a finite-sized system, the wave function, energy, and consequently the entropy of a block will vary continuously in any finite-sized system. In this case, the level crossing develops only asymptotically, and the jump in the entropy appears only in the $N \rightarrow \infty$ limit.

In this work, we consider (i) $s_{l}$, the entropy of the $l^{\text {th }}$ rung, (ii) $s_{l, l+1}$, the two-rung entropy of the neighboring $l^{\text {th }}$ and $(l+1)^{\text {st }}$ rungs, (iii) $s_{l, l+1}^{(i)}$, the two-site entropy of the spins $\mathbf{S}_{l}^{(i)}$ and $\mathbf{S}_{l+1}^{(i)}$ on chain $i$, and also (iv) $s_{N}(l)$, the entropy of a block formed by the left $l$ rungs of a ladder with $N$ rungs. To avoid end effects, we compute $s_{l}$, $s_{l, l+1}$, and $s_{l, l+1}^{(i)}$ in the middle of the ladder, for $l=N / 2$ or $l=N / 2+1$. As discussed above, one of our primary interests is to identify dimerized phases which may arise and the concomitant breaking of translational symmetry. The appearance of a columnar dimerized phase can be detected by considering the difference of two-rung entropies

$$
D_{s}=s_{l+1, l+2}-s_{l, l+1} \quad l=N / 2 \text {. }
$$

Alternatively, taking the block entropy $s_{N}(l)$ of the left $l$ rungs, one can consider

$$
\tilde{D}_{s}=s(l)-s(l+1) \quad l=N / 2
$$

which tells how the block entropy of the left half changes when an extra rung is added. The appearance of staggered dimerization can be detected by considering the difference of two-site entropies on the two chains

$$
P_{s}=\left(s_{l+1, l+2}^{(1)}-s_{l, l+1}^{(1)}\right)-\left(s_{l+1, l+2}^{(2)}-s_{l, l+1}^{(2)}\right) \quad l=N / 2 .
$$

Further information about the phases that arise can be deduced by studying the length dependence of $s_{N}(l) \stackrel{58,73,74,75,76}{5}$ For noncritical, gapped models, this quantity saturates to a finite value when $l$ is far from the boundaries, while for critical systems

$$
s_{N}(l)=\frac{c}{6} \ln \left[\frac{2 N}{\pi} \sin \left(\frac{\pi l}{N}\right)\right]+g,
$$

where $c$ is the central charge $\stackrel{58.77}{ }$ Moreover, if the system's ground state is spatially inhomogeneous, oscillations appear in $s_{N}(l) .{ }^{45}$ Hence, the Fourier spectrum

$$
\tilde{s}(k)=\frac{1}{N} \sum_{l=0}^{N} e^{-i k l} s_{N}(l)
$$

carries information 45 about the spatial inhomogeneity: if the amplitude of a peak at a nonzero wave number $k^{*}$ remains finite in the thermodynamic limit, this indicates a periodic spatial modulation of the ground state with wavelength $\lambda=2 \pi / k^{*}$.

In what follows, we will often need to know the large- $N$ behavior of various quantities. For any quantity $A$, the finite-size-scaling Ansatz

$$
A(N)=A_{0}+a / N^{\beta}
$$

has been used, where $A_{0}, a, \beta$ are free parameters determined by a least-squares fitting procedure.

\section{B. Results and Discussion}

\section{Zigzag ladder}

We first present results for the zigzag ladder. Since the phase diagram and properties of this model are well known, it serves as a test case for the other models. The $J_{\perp}, J_{2}$ parameter space was explored by calculating various entropy functions for $J_{\perp}$ and $J_{2}$ satisfying

$$
J_{\perp}+J_{2}=C J_{\|}
$$

for several values of $C$. In the numerical calculations the energy scale was set by taking $J_{\|}$to be unity.

We first show results obtained for $C=2$. As seen in Fig. 11] $s_{l}$ in the middle of the ladder is discontinuous at $J_{\perp}=J_{2}=J_{\|}$, indicating a first-order transition. Besides the discontinuity, the inset of Fig. 11 shows a minimum in the entropy at $J_{2} \simeq 1.36 J_{\|}$, indicating a possible second transition. Moreover, there is also a minimum in $\tilde{s}(k)$ at an incommensurate $k^{*}$ in the region $0.5<J_{2} / J_{\|}<1.36$. However, on physical grounds, there is no reason to expect a second transition in the zigzag ladder since any deviation from the $J_{\perp}=J_{2}$ line drives the system into the rung-singlet or Haldane phase. Indeed, this minimum is a finite-size effect — it originates from the two end spins of the Haldane phase. To substantiate this, notice that there is no sign of this minimum in the rungsinglet phase, while we know that the phase boundaries must be symmetric under the interchange of $J_{\perp}$ and $J_{2}$. Furthermore, by attaching spin-1/2's to the ends of the 
ladder with a strong anitferromagnetic coupling, the end spins can be eliminated ${ }^{78}$ When the calculations are repeated with these extra spin-1/2's attached to the ends, the minimum in $\tilde{s}(k)$ at an incommensurate $k^{*}$ disappears.

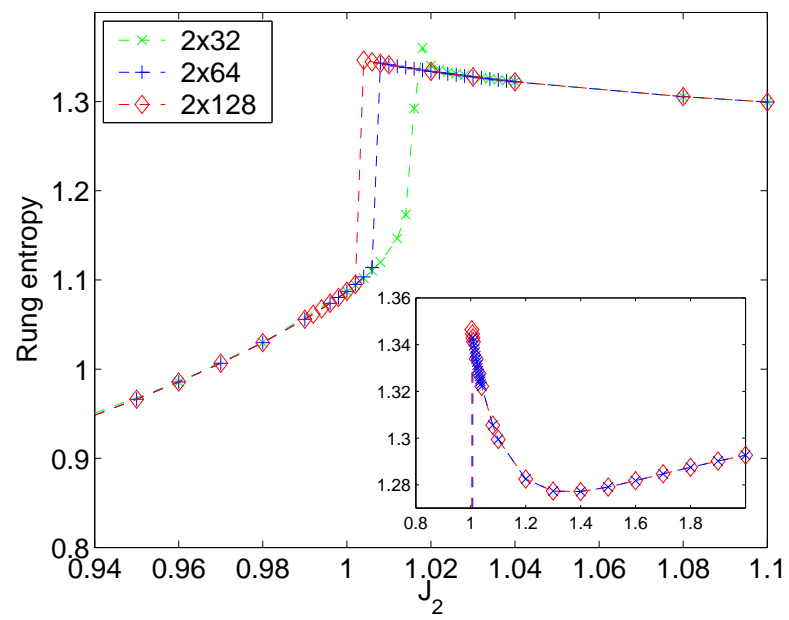

FIG. 11: (Color online) Single-rung entropy as a function of $J_{2}$ for $J_{\perp}+J_{2}=2 J_{\|}$with $J_{\|}=1$ for three different lengths of the ladder.
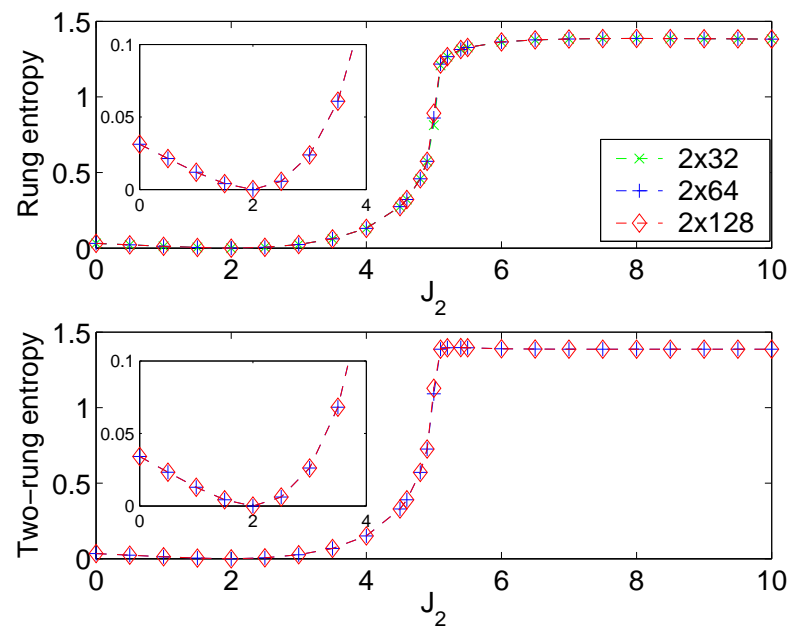

FIG. 12: (Color online) Single-rung and block entropies for $J_{\perp}+J_{2}=10 J_{\|}$, as a function of $J_{2}$ for ladders with 32,64 and 128 rungs. The insets show the behavior near $J_{2}=2 J_{\|}$, where the entropies vanish.

Performing the calculations for couplings satisfying (52) with other values of $C$, a behavior similar to Fig. 11 was observed whenever the line $J_{\perp}=J_{2}$ was crossed at $J_{\perp}<J_{\|} / 0.241$. That indicates that the system undergoes a first-order transition (along the line $J_{\perp}=J_{2}$ ) for $J_{\perp}<J_{\|} / 0.241$. Different behaviors in the entropies were observed, however, for stronger interchain couplings. Fig. 12 shows $s_{l}$ (top panel) and $s_{l, l+1}$ (bottom panel) in the middle of the ladder for $J_{\perp}+J_{2}=10 J_{\|}$. In the rung-singlet phase $s_{l}$ is small, as the spins on a rung are predominantly in a singlet state; in the Haldanelike phase $s_{l}$ is close to $\ln 4 \simeq 1.386$, since the valence bonds are formed predominantly between neighboring rungs. Notice, however, that $s_{l}$ no longer has a jump at $J_{\perp}=J_{2}=C J_{\|} / 2$ (as it did in Fig. 111); rather, $s_{l}$ and $s_{l, l+1}$ have a discontinuity in their slope. When $s_{N}(l)$ was computed at the point $J_{\perp}=J_{2}$, it was found that it could be fit well with the form given in (49) with $c=1$. These results are consistent with a continuous transition between the rung-singlet and Haldane-like phases in this regime. They are consistent with the fact that, if the zigzag ladder is written as a single chain with nearestand next-nearest-neighbor couplings, the chain is critical in this regime with the low-energy physics being described by (24).

The insets of Fig. 12 show $s_{l}$ and $s_{l, l+1}$ in a region about $J_{2}=2 J_{\|}$; we see that the entropy functions vanish at $J_{2}=2 J_{\|}$for any length of the ladder. This is because the exact ground state is a product of rung singlets [see Eq. (15)] for $J_{2}=2 J_{\|}$. In fact, the entropies vanishes along the entire line $J_{2}=2 J_{\|}$when $J_{\perp} \geq 2 J_{\|}$, as (15) is the exact ground state. Furthermore, computing the entropy of the two spins coupled along the diagonal by $J_{2}, \mathbf{S}_{l}^{(1)}$ and $\mathbf{S}_{l+1}^{(2)}$, this entropy was found to vanish for $J_{\perp}=2 J_{\|}$when $J_{2} \geq 2 J_{\|}$. This is because (20) is the exact ground state along this line. More generally, due to the symmetry of the model, one obtains the same results presented in Figs. 11 and 12 if $J_{\perp}$ and $J_{2}$ are interchanged; instead of considering rung entropies, one must consider the entropies of diagonally coupled spins.

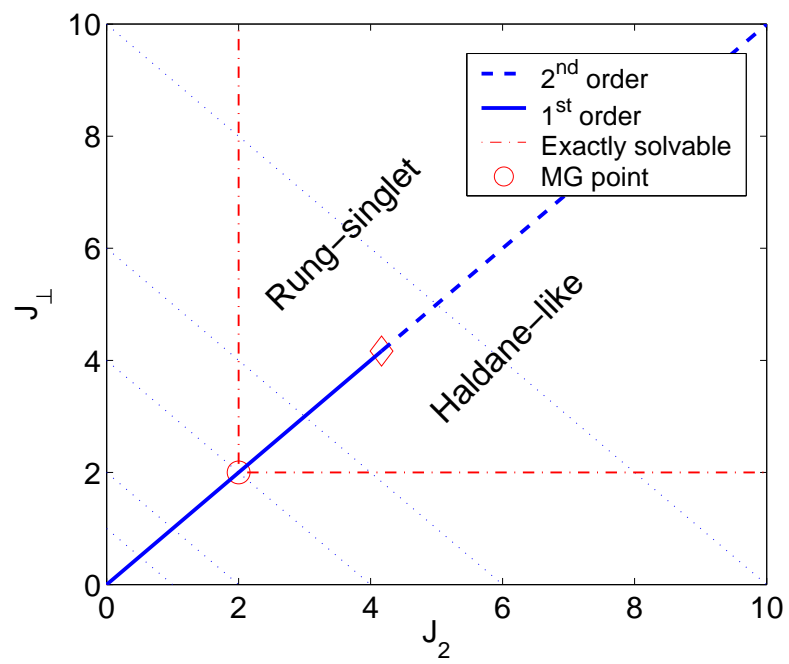

FIG. 13: (Color online) Phase diagram of the zigzag ladder. The first- and second-order transition between the rungsinglet and Haldane phases are denoted by solid and dashed lines, respectively. The ground state along the dashed-dotted line is an exact rung-singlet or diagonal-singlet valence-bond state. The dotted lines indicate the parameter values wher the DMRG calculation were done.

Putting together these results, we arrive at the phase diagram in Fig. 13. The transition line at $J_{\perp}=J_{2}$ is 
of first order in the weak-coupling limit, which becomes of second order at strong couplings. The entire region above (below) the line $J_{\perp}=J_{2}$ is continuously related to the exactly solvable line $J_{2}=2 J_{\|}\left(J_{\perp}=2 J_{\|}\right)$and, hence, is in the rung-singlet (Haldane) phase. In terms of the effective low-energy Hamiltonian discussed in the previous section, the physics in these phases is determined by the $\mathbf{n}_{1} \cdot \mathbf{n}_{2}$ term; in the rung-singlet (Haldane) phase, its coefficient is positive (negative). As discussed in Sec. IV, the rung-singlet (Haldane) phase has $Q=$ even ( $Q=$ odd) topological order, and the elementary excitations are gapped magnons with spinons being confined. Along the $J_{\perp}=J_{2}$ line, the two topologically distinct ground states become degenerate, spinons are deconfined, being domain walls between the two topologically distinct (and energetically degenerate) ground states. As mentioned in the previous section, at weak coupling the physics is determined by the $\left(\mathbf{J}_{1 R} \cdot \mathbf{J}_{2 L}+\mathbf{J}_{2 R} \cdot \mathbf{J}_{1 L}\right)$ term (with the $\mathbf{n}_{1} \cdot \partial_{x} \mathbf{n}_{2}$ and $\epsilon_{1} \partial_{x} \epsilon_{2}$ terms giving rise to incommensuration). Looking at Eq. (40), this fractionalized phase occurs because the geometry of the zigzag ladder fine tunes away an infinite number of operators which could cause dimerization.

\section{Cross-Coupled Ladder}

As with the zigzag ladder, we calculated $s_{l}$ and $s_{l, l+1}$ for $J_{\perp}$ and $J_{\mathrm{X}}$ satisfying (52) with various values of $C$. Fig. 14 shows results obtained for intermediate values of the interchain couplings, $C=2$ and $C=3$. Here, we see the entropy functions display a finite jump, indicating a first-order transition. The inset shows that $s_{l}$ vanishes (independent of the ladder's length) at $J_{\mathrm{X}}=J_{\|}$. This occurs because (15) is the exact ground state for $J_{\mathrm{X}}=J_{\|}$ with $J_{\perp} \geq 2 J_{\|}$. It was shown in Ref. 32 that (15) is, in fact, the ground state for $J_{\mathrm{X}}=J_{\|}$and $J_{\perp} \geq 1.401 J_{\|}$. Our numerical results are in agreement with this prediction.

Besides the finite jump at $J_{\mathrm{X}}=0.8$, Fig. 14 also shows that the two-site entropy possesses a minimum around $J_{\mathrm{X}} \approx 1$; furthermore, weak incommensurate oscillations were found to appear in $s_{N}(l)$. Similar to the zigzag ladder, these are finite-size effects due to the end spins in the Haldane phase. Indeed, when calculations are repeated for a system in which spin-1/2's are attached to the ends of the ladder (to freeze the end spins), the block entropy $s_{N}(l)$ saturates for shorter chains and the minimum in $\tilde{s}(k)$ at an incommensurate $k^{*}$ disappears. The amplitude of the remaining negative peak in $\tilde{s}(k)$ at $k^{*}=\pi$ was found to vanish in the thermodynamic limit. Hence, no spatial inhomogeneity develops along the transition line.

The numerical results show different behavior when $C<1.3 . s_{l}$ as a function of $J_{\mathrm{X}}$ is shown in Fig. 15 for $C=1$. The ground-state wave function is continuous in the weak-coupling regime. As longer and longer ladders are considered, $s_{l}$ exhibits a sharper and sharper

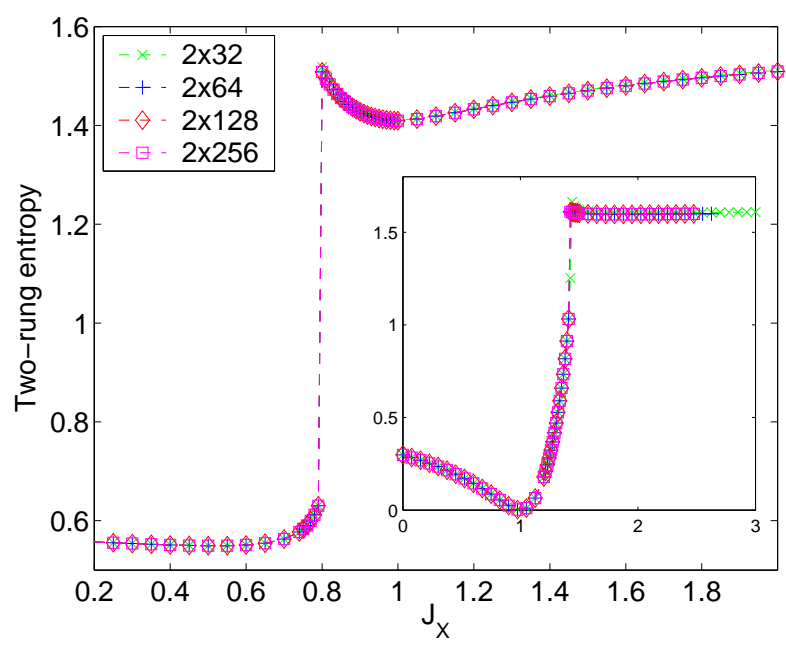

FIG. 14: (Color online) Two-rung entropy of the crosscoupled ladder as a function of $J_{\mathrm{X}}$ for $J_{\perp}=2 J_{\|}-J_{\mathrm{X}}$ for various system sizes. The dashed line is a guide to the eyes. The inset shows the same but for the $J_{\perp}=3 J_{\|}-J_{\mathrm{X}}$.

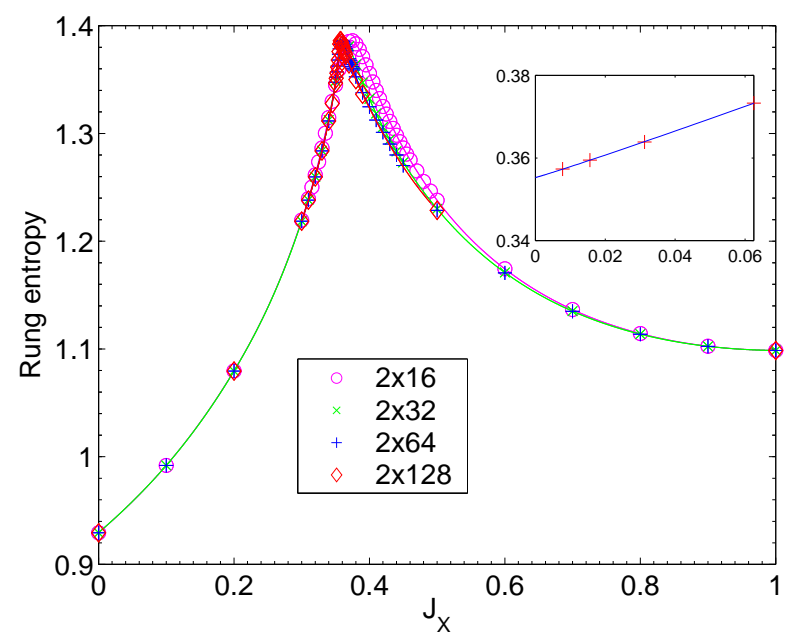

FIG. 15: (Color online) One-rung entropy of the cross-coupled ladder model for $J_{\perp}+J_{\mathrm{X}}=J_{\|}$, for several system sizes. The solid line is a polynomial fit. The inset shows the finite-size scaling of the position of the maximum of the entropy.

maximum (bounded from above by $\ln 4$ ), developing into a cusp at $J_{\mathrm{X} c} / J_{\|}=0.355(3)$. Such behavior is suggestive of a continuous transition. Recent numerical works reported a continuous transition in the weak-coupling regime, $\stackrel{20,33}{ }$ in disagreement with analytic results $\frac{10}{\underline{10}}$ as well as previous numerical calculations 11 However, the analytic results are expected to be reliable in the weakcoupling regime. As was checked by the DBSS procedure, a reliable extrapolation of the gap requires calculations on longer ladders and keeping a significantly larger number of block states than was available in Ref. 20. The same holds for the entropy. Moreover, as was shown above, spurious effects can arise due to end spins. When the calculations were repeated by attaching spin- $1 / 2$ 's to 
the ends of the ladder; $s_{l}$ behaves somewhat differently. As shown in Fig. 16, instead of an abrupt change in slope, a jump now seems to develop; its position scales to the same value of $J_{\mathrm{X} c}$ obtained in Fig. 15. This behavior suggests that the transition is of first-order. Based on these considerations, we believe the transition is, in fact, firstorder. Although the singlet ground-state wave function is continuous in the weak-coupling regime for finite-sized systems, a crossing with the next singlet level (which we found to be at relatively high energy for weak couplings, far from the exactly solvable line) may develop in the $N \rightarrow \infty$ limit, and the asymmetric cusp in Fig. 15 may develop into a jump.

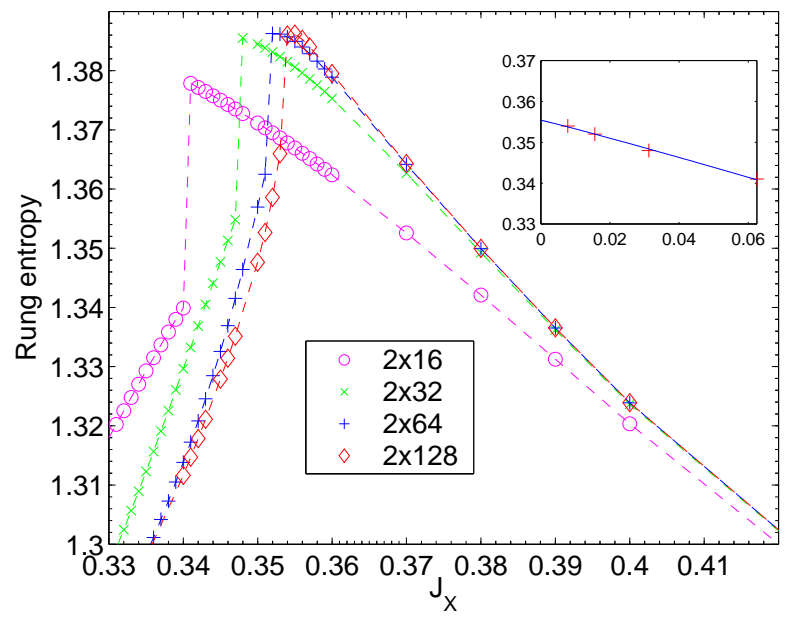

FIG. 16: (Color online) Same as Fig. 15 but with spin-1/2 particles attached to the two ends of the ladder with strong antiferromagnetic couplings. The inset shows the finite-size scaling of the position of the jump in the entropy.

A further interesting feature of the model for weak interchain coupling is shown in Fig. 17, More specifically, the two-rung entropy $s_{l, l+1}$ measured for $l=N / 2$ exhibits two well separated peaks at $J_{\mathrm{X}_{1}}(N)$ and $J_{\mathrm{X} c_{2}}(N)$, but it exhibits a single peak for $l=N / 2+1$. The difference $D_{\mathrm{s}}(N)$ is also finite in the region between $J_{\mathrm{X} c_{1}}$ and $J_{\mathrm{X}_{c_{2}}}$. This could suggests the existence of a columnar dimer phase in a narrow range of couplings, as predicted in Ref. 12. However, the two peaks merge in the $N \rightarrow \infty$ limit and the width of the putative dimer phase shrinks to zero. As shown in the inset of Fig. 17 a finite-size scaling analysis gives the same critical value as the one-rung entropy. The same behavior was observed in the calculation along the line $J_{\mathrm{X}}=0.2 J_{\|}$confirming the findings of Ref. 33 .

We have also computed $s_{N}(l)$ and its Fourier transform $\tilde{s}(k)$. $|\tilde{s}(k)|$ was found to have an extra peak at $k^{*}=\pi$, besides the one at $k=0$. However, $\left|\tilde{s}\left(k^{*}=\pi\right)\right|$ was found to vanish in the large- $N$ limit, indicating that the ground state is always spatially homogeneous. Similar behavior was found for other values of $J_{\perp}$ and $J_{\mathrm{X}}$ in the weak-coupling regime. Furthermore, we computed the staggered dimerization [see Eq. (48)] and we found that

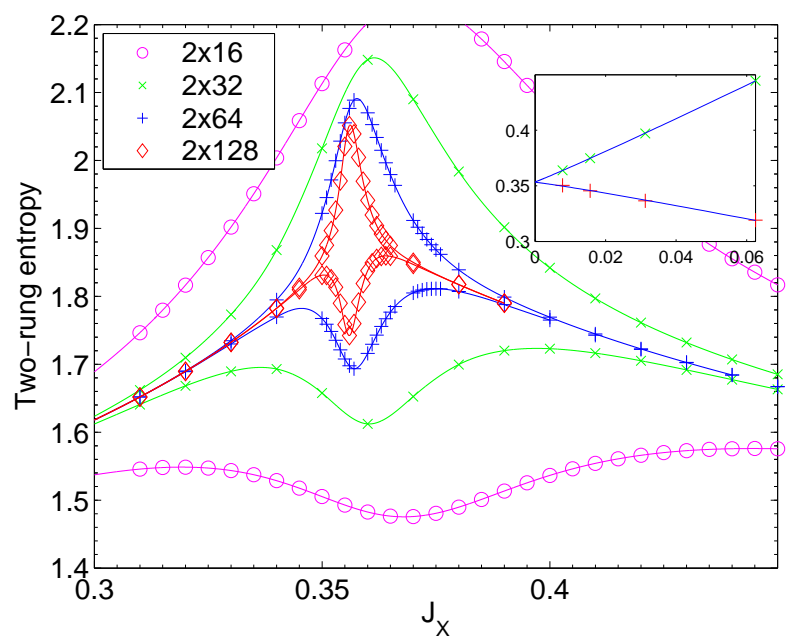

FIG. 17: (Color online) Two-rung entropy of the crosscoupled ladder model for $J_{\perp}+J_{\mathrm{X}}=J_{\|}$for various system sizes. The solid line is a polynomial fit. The inset shows the finite-size scaling of the positions of the peaks of the entropy.

it also vanishes in the $N \rightarrow 0$ limit. This provides strong evidence that, at least for weak interchain coupling, there is no intermediate columnar or staggered dimer phase between the rung-singlet and Haldane phases.

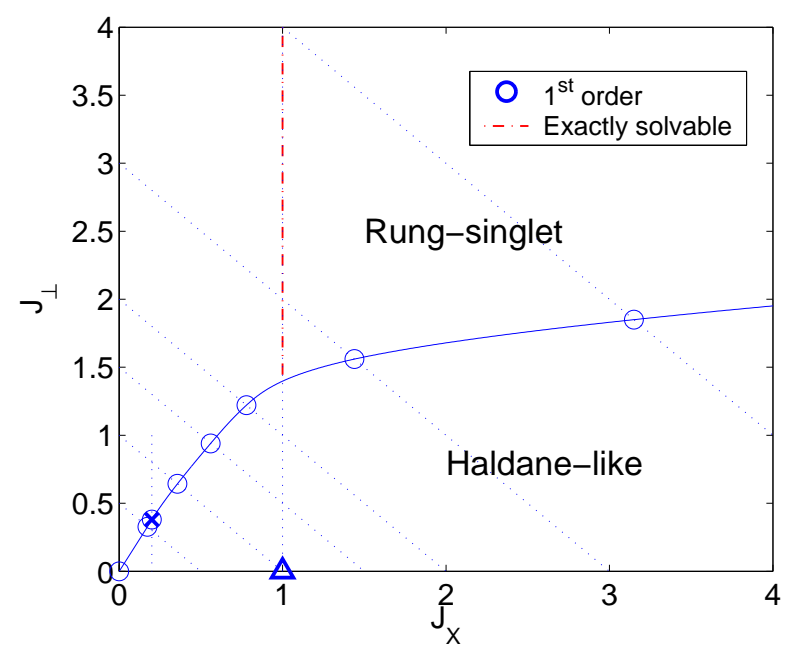

FIG. 18: (Color online) Phase diagram of the cross-coupled ladder. The symbol $\times$ denotes the transition point calculated in Ref. 33. $\triangle$ denotes the point where the ladder model is equivalent to a spin-1 chain. The ground state is an exact rung-singlet state along the dashed-dotted line.

Putting together these results, we obtain the phase diagram for the cross-coupled ladder, shown in Fig. 18 , The entire region above the transition line is continuously related to the exactly solvable line $J_{\mathrm{X}} / J_{\|}=1$ and, hence, is in the rung-singlet phase. The entire region below the transition line is continuously related the point $J_{\mathrm{X}}=1, J_{\perp}=0$ and, hence, is in the Haldane phase. As with the zigzag ladder, the physics in these phases is 
determined by the $\mathbf{n}_{1} \cdot \mathbf{n}_{2}$ term in the low-energy Hamiltonian - these phases have topological order, and confined spinons. As discussed in the previous section, at the transition there is a subtle competition between the $\left(\mathbf{J}_{1 R} \cdot \mathbf{J}_{2 L}+\mathbf{J}_{1 L} \cdot \mathbf{J}_{2 R}\right)$ and $\epsilon_{1} \epsilon_{2}$ terms, which give rise to a fractionalized and dimerized phase, respectively. However, the entire transition line appears to be of first-order, with no evidence for an intermediate dimerized phase being found. Hence, our results suggest that a dimerized phase does not appear in this model. In constructing the phase diagram, we made use of a duality relationship of the model. Similar to what was described for the diagonal ladder in Sec. II, one can interchange the spins on every second rung: $\mathbf{S}_{2 l}^{1} \leftrightarrow \mathbf{S}_{2 l}^{2}$. When this is done for this ladder model, another cross-coupled ladder is obtained but with $J_{\|}$and $J_{\mathrm{X}}$ interchanged. This implies that energies and, in particular, energy gaps satisfy

$$
E\left(J_{\|}, J_{\perp}, J_{\mathrm{X}}\right)=E\left(J_{\mathrm{X}}, J_{\perp}, J_{\|}\right) .
$$

Scaling by $J_{\|}$, one obtains

$$
E\left(J_{\perp} / J_{\|}, J_{\mathrm{X}} / J_{\|}\right)=\left(J_{\mathrm{X}} / J_{\|}\right) E\left(J_{\perp} / J_{\mathrm{X}}, J_{\|} / J_{\mathrm{X}}\right) .
$$

\section{Diagonal Ladder}

As with the other models, we computed $s_{l}$ and $s_{l, l+1}$ for $J_{\perp}$ and $J_{2}$ satisfying (52) with various values of $C$. Here, in contrast to the other two models, we find a dimerized phase intervening between the rung-singlet and Haldane phases.

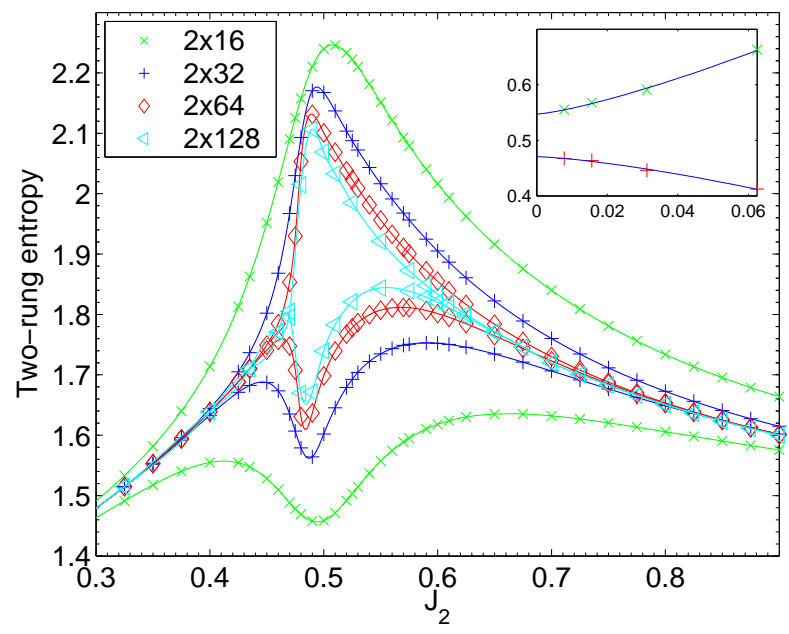

FIG. 19: (Color online) Two-rung entropy of the diagonal ladder model for $J_{\perp}+J_{2}=J_{\|}$for various system sizes. The solid line is a polynomial fit. The inset shows the finite-size scaling of the positions of the peaks of the entropy.

Fig. 19 shows $s_{l, l+1}$ for $J_{\perp}+J_{2}=J_{\|}$. While the behavior appears similar to Fig. [17, the $N \rightarrow \infty$ limit is drastically different. Indeed, while a single transition was found for the cross-coupled ladder, the inset of
Fig. 19 shows the two peaks do not collapse in this model. Hence, Fig. 19 suggests the system undergoes two distinct transitions at $J_{2 c_{1}} / J_{\|}=0.459$ and $J_{2 c_{2}} / J_{\|}=0.563$, with a columnar dimerized phase between the two transition lines. To substantiate this, we have computed $s_{N}(l)$ and subsequently $\left|\tilde{s}\left(k^{*}=\pi\right)\right|$, the entropy difference between neighboring plaquettes $D_{s}$ (shown in Fig. 20), and also the energy difference between neighboring plaquettes. All of these quantities were found to scale to a finite value in the region between $J_{2 c_{1}}$ and $J_{2 c_{2}}$. Similar behavior was found for other values of the parameters in the weak-coupling limit. Hence, we conclude a columnar dimerized phase does, in fact, exist between the rungsinglet and Haldane phases in this model.
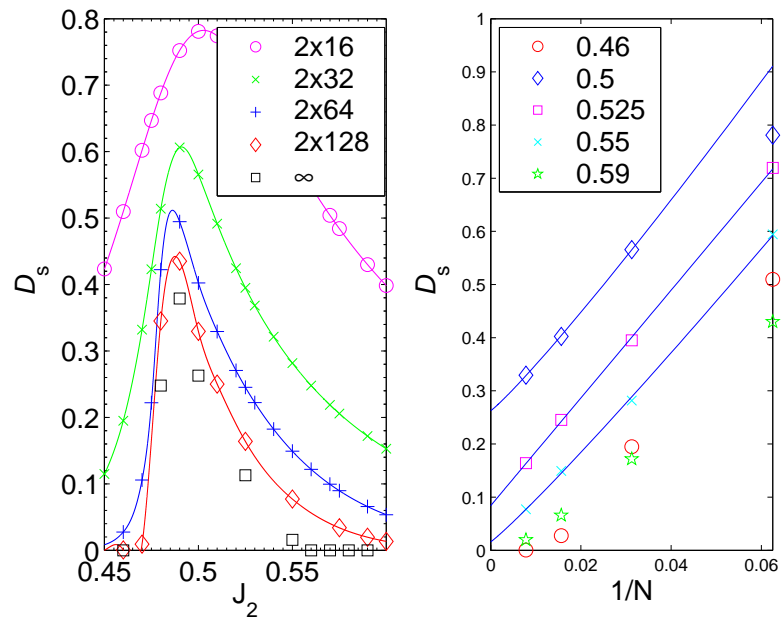

FIG. 20: (Color online) The dimerization entropy of the diagonal ladder model for $J_{\perp}+J_{2}=J_{\|}$for various system sizes. The solid line is a polynomial fit. The left panel shows the finite-size scaling of the $D_{\mathrm{s}}$ for various $J_{2}$ values.

When the calculations were repeated for $J_{\perp}+J_{2}>2 J_{\|}$, a single first-order transition was obtained. It is worth noting that due to the rather small values of the gap, the asymptotic behavior can be seen only for long ladders. As before, convergence can be accelerated in the Haldane phase by attaching spin- $1 / 2$ 's to the ends of the ladder (to pin the end spins).

Fig. 21 summarizes our finding for the phase diagram of the diagonal ladder. The entire region above the transition line is continuously related to the exactly solvable line $J_{2} / J_{\|}=2$ and, hence, is in the rung-singlet phase. The entire region below the transition line is in the Haldane phase. As with the previous two ladder models, these phases have topological order with confined spinons; the physics in these phases is determined by the $\mathbf{n}_{1} \cdot \mathbf{n}_{2}$ term in the low-energy Hamiltonian. However, contrary to what was found for the cross-coupled and zigzag ladders, from the discussion in the previous section, it is reasonable to expect a regime in between the rung-singlet and Haldane phases where the diagonal ladder dimerizes (i.e., where the physics is determined by the $\epsilon_{1} \epsilon_{2}$ term). This is, indeed, found to be the case - a 


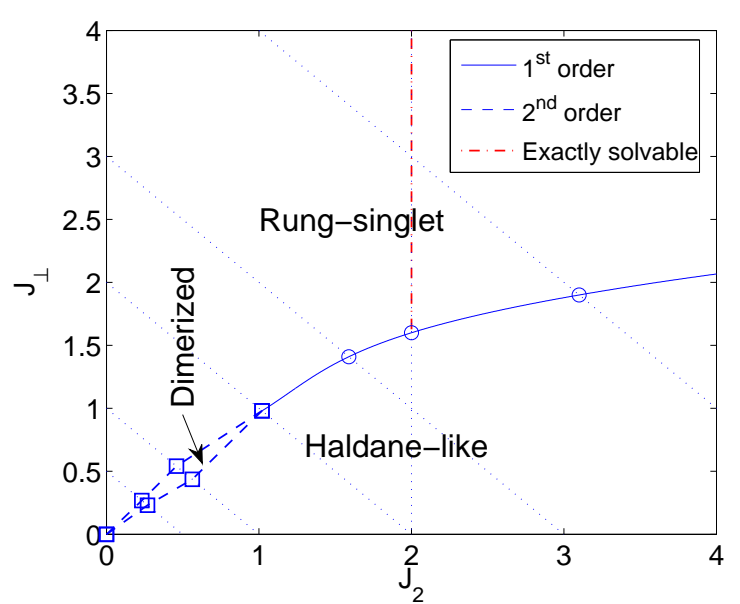

FIG. 21: (Color online) Phase diagram of the diagonal ladder. The first and second-order phase transition points are indicated by the circle and square symbols, respectively. The exact rung-singlet ground state is indicated by a dashed-dotted line.

narrow, but extended region is found where the ground state is dimerized. The first order transition is replaced in the weak coupling regime by two second-order transitions.

\section{CONCLUDING REMARKS}

In this work we considered several frustrated spin ladder models, which are related to higher-dimensional models of current interest. In large regions of parameter space, these models have short-range RVB ground states with topological order; they are adiabatically related to the ground states of the rung-singlet or Haldane phase. We investigated the role of frustrating interactions on the models, addressing in particular how the transition between phases with different topological order occurs. In the simplest case, a direct transition takes place along the line where the even- and odd-topology phases become degenerate. While the elementary excitations of the topologically ordered phases are gapped magnons, spinons become deconfined along the transition line and a fractionalized phase is obtained. Alternatively, the transition may occur in two steps, with an intermediate phase having broken translational symmetry. In this case, spinons always remain confined.

An important observation from our analysis is the strong "desire" for broken-symmetry phases to arise at the transition between the rung-singlet and Haldane phases. Indeed, we saw that spin models typically give rise to an infinite number of operators that could cause dimerization 12 These operators arose in the diagonal ladder, and a dimerized phase was seen to appear in the phase diagram. These operators also arose in the crosscoupled ladder; nevertheless, in agreement with Ref. 33, we found no evidence for dimerized phases in our numerics. The RG equations suggest that this occurs due to a subtle interplay/competition of quantum fluctuations. Although no dimerized phase appears in the crosscoupled ladder studied in this paper, in this delicate situation even small perturbations are likely to drive the cross-coupled model into dimerized phases as shown in Ref. 79. The zigzag ladder was an exception - the model's geometry fine-tunes away the infinite number of operators which could cause dimerization.

We believe these results give an outlook into the physics of higher-dimensional systems. In particular, our results show the importance of a system's geometry in achieving the necessary liquidity for fractionalized excitations to occur. Hence, our results illustrate why fractionalized phases are hard to come by — fractionalized phases are delicate objects, requiring some level of fine-tuning. Even on lattices where the necessary fine-tuning occurs (such as the triangular lattice), small perturbations due to, e.g., spin-phonon coupling 51 or ring exchanges are likely to drive the system into a dimerized phase.

\section{Acknowledgements}

EHK gratefully acknowledges the warm hospitality of the Research Institute for Solid State Physics and Optics (Budapest, Hungary), where parts of this work were performed. This work was supported by the NSERC of Canada (EHK), a SHARCNET Research Chair (EHK), a SHARCNET Senior Visiting Scholar Award (EHK and JS), and the Hungarian Research Fund OTKA Grant Nos. K 68340, F 46356, and NF 61726 (OL and JS).
${ }^{1}$ For reviews, see Frustrated Spin Systems, edited by H. T. Diep (World Scientific, Singapore 2004); Quantum Magnetism, Lect. Notes Phys. 645 (2004).

2 P. W. Anderson, Science 235, 1196 (1987).

3 S. Sachdev, in Low Dimensional Quantum Field Theory for Condensed Matter Physicists, edited by Y. Lu, S. Lundqvist, and G. Morandi (World Scientific, Singapore 1995).

4 E. Fradkin, Field Theories of Condensed Matter Systems
(Addison-Wesley, New York 1991).

5 N. Bonesteel, Phys. Rev. B 40, 8954 (1989).

6 X. G. Wen, Phys. Rev. B 44, 2664 (1991); T. Senthil and M. P. A. Fisher, Phys. Rev. B 61, 9690 (2000).

7 R. Moessner, S. Sondhi, and E. Fradkin, Phys. Rev. B 65, 24504 (2001).

8 F. D. M. Haldane, Phys. Rev. Lett. 61, 1029 (1988); N. Read and S. Sachdev, Phys. Rev. B 42, 4568 (1990).

9 S. R. White, Phys. Rev. B 53, 52 (1996). 
10 E. H. Kim, G. Fáth, J. Sólyom, and D. J. Scalapino, Phys. Rev. B 62, 14965 (2000).

11 G. Fáth, Ö. Legeza, and J. Sólyom, Phys. Rev. B 63, 134403 (2001).

12 O. A. Starykh and L. Balents, Phys. Rev. Lett. 93, 127202 (2004).

13 D. Allen, F. H. Essler, and A. A. Nersesyan, Phys. Rev. B 61, 8871 (2000).

14 R. M. Noack, S. R. White, and D. J. Scalapino, Physica C 270, 281 (1996); H. J. Schulz, Phys. Rev. B 53, R2959 (1996); L. Balents and M. P. A. Fisher, Phys. Rev. B 53, 12133 (1996).

15 A. Seidel and D.-H. Lee, Phys. Rev. Lett. 97, 56804 (2006).

16 For a review, see E. Dagotto, Rep. Prog. Phys. 62, 1525 (1999).

17 D. J. Scalapino, Nature 377, 12 (1995); E. Dagotto and T. M. Rice, Science 271, 618 (1996).

18 S. R. White, R. M. Noack, and D. J. Scalapino, Phys. Rev. Lett. 73, 886 (1994).

19 D. G. Shelton, A. A. Nersesyan, A. M. Tsvelik, Phys. Rev. B 53, 8521 (1996).

20 X. Wang, Mod. Phys. Lett. B 14, 327 (2000).

21 Ö. Legeza, G. Fáth, and J. Sólyom, Phys. Rev. B 55, 291 (1997).

22 Ö. Legeza and J. Sólyom, Phys. Rev. B 56, 14449 (1997).

${ }^{23}$ F. D. M. Haldane, Phys. Rev. Lett. 50, 1153 (1983); Phys. Lett. 93A, 464 (1983).

24 J. Sólyom and J. Timonen, Phys. Rev. B 38, 6832 (1988).

25 Y. Xian, Phys. Rev. B 52, 12485 (1995).

26 G. Sierra, M. A. Martín-Delgado, S. R. White, D. J. Scalapino, and J. Dukelsky, Phys. Rev. B 59, 7973 (1999); M. A. Martín-Delgado, J. Rodriguez-Laguna, and G. Sierra, ibid. 72, 104435 (2005).

27 S. Wakimoto, G. Shirane, Y. Endoh, K. Hirota, S. Ueki, K. Yamada, R. J. Birgeneau, M. A. Kastner, Y. S. Lee, P. M. Gehring, and S. H. Lee, Phys. Rev. B 60, R769 (1999); S. Wakimoto, R. J. Birgeneau, M. A. Kastner, Y. S. Lee, R. Erwin, P. M. Gehring, S. H. Lee, M. Fujita, K. Yamada, Y. Endoh, K. Hirota, and G. Shirane, Phys. Rev. B 61 , 3699 (2000).

28 J. M. Tranquada, D. J. Buttrey, V. Sachan, and J. E. Lorenzo, Phys. Rev. Lett. 73, 1003 (1994).

29 V. Sachan, D. J. Buttrey, J. M. Tranquada, J. E. Lorenzo, and G. Shirane, Phys. Rev. B 51, 12742 (1995).

30 M. P. Gelfand, Phys. Rev. B 43, 8644 (1991).

31 J. S. Gardner, S. R. Dunsiger, B. D. Gaulin, M. J. P. Gingras, J. E. Greedan, R. F. Kiefl, M. D. Lumsden, W. A. MacFarlane, N. P. Raju, J. E. Sonier, I. Swainson, and Z. Tun, Phys. Rev. Lett. 82, 1012 (1999); A. S. Wills, N. P. Raju, and J. E. Greedan, Chem. Mater. 11, 1510 (1999); M. J. Harris, M. P. Zinkin, Z. Tun, B. M. Wankly, and I. P. Swainson, Phys. Rev. Lett. 73, 189 (1994); R. De Pape and G. Ferey, Mater. Res. Bull. 21, 971 (1986).

32 Z. Weihong, V. Kotov, and J. Oitmaa, Phys. Rev. B 57, 11439 (1998)

33 H.-H. Hung, C.-D. Gong, Y.-C. Chen, and M.-F. Yang, Phys. Rev. B. 73, 224433 (2006).

34 R. Coldea, D. A. Tennant, A. M. Tsvelik, and Z. Tylczynski, Phys. Rev. Lett. 86, 1335 (2001); R. Coldea, D. A. Tennant, and Z. Tylcznski, Phys. Rev. B 68, 134424 (2003).

35 M. Matsuda and K. Katsumata, J. Magn. Magn. Mater. 140-145, 1671 (1995); Z. Hiroi, M. Azuma, M. Takano, and Y. Bando, J. Solid State Chem. 95, 230(1991); N. Motoyama, H. Eisaki, and S. Uchida, Phys. Rev. Lett. 76, 3212 (1996); M. Matsuda, K. Katsumata, K. M. Kojima, M. Larkin, G. M. Luke, J. Merrin, B. Nachumi, Y. J. Uemura, H. Eisaki, N. Motoyama, S. Uchida, and Z. Tylczynski, Phys. Rev. B 79, 151 (1997).

${ }^{36}$ K. Okamoto and K. Nomura, Phys. Lett. A 169, 433 (1993).

37 S. Eggert, Phys. Rev. B 54, R9612 (1996).

38 F. D. M. Haldane, Phys. Rev. B 25, R4925 (1982); ibid. 26, 5257(E) (1982)

39 C. K. Majumdar and D. K. Ghosh, J. Math. Phys. 10, 1388 (1969); ibid. 10, 1399 (1969).

40 A. K. Kolezhuk and H.-J. Mikeska, Phys. Rev. B 56, R11380 (1997).

41 T. Tonegawa and I. Harada, J. Phys. Soc. Jpn. 56, 2153 (1987); T. Tonegawa, I. Harada, and M. Kaburagi, J. Phys. Soc. Jpn. 61, 4665 (1992).

42 R. Chitra, S. Pati, H. R. Krishnamurthy, D. Sen, and S. Ramasesha, Phys. Rev. B 52, 6581 (1995).

43 S. R. White and I. Affleck, Phys. Rev. B 54, 9862 (1996).

44 A. A. Aligia, C. D. Batista, and F. H. L. Essler, Phys. Rev. B 62, 3259 (2000).

45 Ö. Legeza, J. Sólyom, L. Tincani, and R. M. Noack, Phys. Rev. Lett. 99, 087203 (2007).

46 B. S. Shastry and B. Sutherland, Phys. Rev. Lett. 47, 964 (1981).

47 I. Bose, Phys. Rev. B 45, 13072 (1992); I. Bose and S. Gayen, Phys. Rev. B 48, 10653 (1993).

48 X.-G. Wen, Quantum Field Theory of Many-Body Systems (Oxford University Press, New York 2004).

49 M. P. M. den Nijs and K. Rommelse, Phys. Rev. B 40, 4709 (1989).

50 Y. Nishiyama, N. Hatano, and M. Suzuki, J. Phys. Soc. Jpn. 64, 1967 (1995).

51 A. A. Nersesyan and A. M. Tsvelik, Phys. Rev. Lett. 78, 3939 (1997).

52 A. A. Nersesyan, A. O. Gogolin, and F. H. L. Essler, Phys. Rev. Lett. 81, 910 (1998).

53 E. Orignac and T. Giamarchi, Phys. Rev. B 56, 7167 (1997).

54 D. C. Cabra, A. Honecker, and P. Pujol, Eur. Phys. J B13, 55 (2000).

55 P. Di Francesco, P. Mathieu, and D. Senechal, Conformal Field Theory (Springer-Verlag, New York 1997).

56 I. Affleck, in Fields, Strings and Critical Phenomena, edited by E. Brézin and J. Zinn-Justin (North Holland, Amsterdam 1990).

57 J. Cardy, Scaling and Renormalization in Statistical Physics, (Cambridge University Press, Cambridge, 1996).

58 P. Calabrese and J. Cardy, J. Stat. Mech.: Theor. Exp. P06002 (2004).

59 S. R. White, Phys. Rev. Lett. 69, 2863 (1992); Phys. Rev. B 48, 10345 (1993).

60 Ö. Legeza, J. Röder, and B. A. Hess, Phys. Rev. B 67, 125114 (2003).

61 Ö. Legeza and J. Sólyom, Phys. Rev. B 70, 205118 (2004).

${ }^{62}$ W. K. Wootters, Phys. Rev. Lett. 80, 2245 (1998).

63 P. Zanardi, Phys. Rev. A 65, 042101 (2002).

64 T. J. Osborne and M. A. Nielsen, Phys. Rev. A 66,032110 (2002).

65 A. Osterloh, L. Amico, G. Falci, and R. Fazio, Nature (London) 416, 608 (2002). 
66 S.-J. Gu, H.-Q. Lin, and Y.-Q. Li, Phys. Rev. A 68, 042330 (2003).

67 J. Vidal, G. Palacios, R. Mosseri, Phys. Rev. A 69, 022107 (2004); J. Vidal, R. Mosseri, J. Dukelsky, ibid. 69, 054101 (2004).

68 S.-J. Gu, S.-S. Deng, Y.-Q. Li, and H.-Q. Lin, Phys. Rev. Lett. 93, 086402 (2004).

69 L.-A. Wu, M. S. Sarandy, and D. A. Lidar, Phys. Rev. Lett. 93, 250404 (2004).

70 M.-F. Yang, Phys. Rev. A 71, 030302(R) (2005).

71 Ö. Legeza and J. Sólyom, Phys. Rev. Lett. 96, 116401 (2006).

72 S.-S. Deng, S.-J. Gu, and H.-Q. Lin, Phys. Rev. B 74 045103 (2006).
73 G. Vidal, J. I. Latorre, E. Rico, and A. Kitaev, Phys. Rev. Lett. 90, 227902 (2003).

74 V. E. Korepin, Phys. Rev. Lett. 92, 096402 (2004).

75 I. Affleck and A. W. W. Ludwig, Phys. Rev. Lett. 67, 161 (1991).

76 N. Laflorencie, E. S. Sørensen, M.-S. Chang, and I. Affleck, Phys. Rev. Lett. 96, 100603 (2006).

77 C. Holzhey, F. Larsen, and F. Wilczek, Nucl. Phys. B424, 443 (1994).

78 S. R. White and D. A. Huse, Phys. Rev. B 48, 3844 (1993).

79 T. Vekua and A. Honecker, Phys. Rev. B. 73, 214427 (2006). 